\begin{tabular}{|c|l|}
\hline Title & Nonlinear stability of stationary solutions for surface diffusion with boundary conditions \\
\hline Author(s) & Garcke, Harald; Ito, Kazuo; Kohsaka, Y oshihito \\
\hline Citation & Hokkaido University Preprint Series in Mathematics, 864, 1-25 \\
\hline Issue Date & 2007 \\
\hline DOI & 10.14943/84014 \\
\hline Doc URL & http://hdl.handle.net/2115/69673 \\
\hline Type & bulletin (article) \\
\hline File Information & pre864.pdf \\
\hline
\end{tabular}

Instructions for use 


\title{
Nonlinear stability of stationary solutions for surface diffusion with boundary conditions
}

\author{
Harald Garcke, Kazuo Ito, and Yoshihito Kohsaka
}

\begin{abstract}
The volume preserving fourth order surface diffusion flow has constant mean curvature hypersurfaces as stationary solutions. We show nonlinear stability of certain stationary curves in the plane which meet an exterior boundary with a prescribed contact angle. Methods include semigroup theory, energy arguments, geometric analysis and variational calculus.
\end{abstract}

Key words. surface diffusion, nonlinear stability, energy method, variational calculus.

AMS subject classifications. 35B35, 35G30, 35K55, 35R35, 53C44.

\section{Introduction}

The surface diffusion flow

$$
V=-\Delta_{S} \kappa
$$

is a geometrical evolution law which describes the surface dynamics for phase interfaces, when mass diffusion only occurs within the interface. Here, $V$ is the normal velocity of the evolving surface, $\Delta_{S}$ is the surface Laplacian, and $\kappa$ is the mean curvature of the surface. The flow (1.1) was first proposed by Mullins [19] in works concerned with thermal grooving. A derivation of (1.1) within rational thermodynamics was given by Davi and Gurtin [6]. In [21], Cahn and Taylor showed that (1.1) is the $H^{-1}$-gradient flow of the area functional, and in [4], Cahn, Elliott, and Novick-Cohen used formal asymptotics to derive (1.1) as the sharp interface limit of the Cahn-Hilliard equation with degenerate mobility. Further, the motion given by (1.1) has the significant geometrical properties that for closed embedded hypersurfaces the enclosed volume is preserved and surface area decreases in time (see e.g. $[7,9]$ ). The evolution law (1.1) leads to a fourth order parabolic equation which is in contrast to the second order mean curvature flow $V=\kappa$. We remark that the mean curvature flow is also area decreasing but changes the enclosed volume.

In this paper we study the motion by surface diffusion for curves in cases where the interface intersects an external boundary. More precisely, we consider the following problem. Let $\Omega$ be an open bounded domain in $\mathbb{R}^{2}$. We look for evolving curves $\Gamma=$ $\left\{\Gamma_{t}\right\}_{t \geq 0}$ lying in $\Omega$ with $\partial \Gamma \subset \partial \Omega$ and satisfying

$$
V=-\kappa_{s s}
$$


for all points on $\Gamma_{t}$ with the boundary conditions

$$
\begin{cases}\Gamma_{t} \perp \partial \Omega & \left(90^{\circ} \text {-angle condition }\right) \\ \kappa_{s}=0 & \text { (no-flux condition })\end{cases}
$$

at $\Gamma_{t} \cap \partial \Omega$, where a subscript $s$ denotes differentiation with respect to the arc-length parameter of the evolving curve $\Gamma_{t}$. The boundary conditions (1.3) are the natural boundary conditions when viewing the flow as the $H^{-1}$-gradient flow of the length functional. It is not difficult to show that under the surface diffusion flow (1.2) with the boundary conditions (1.3) the areas enclosed by $\Gamma_{t}$ and $\partial \Omega$ is preserved and the length of $\Gamma_{t}$ decreases in time. We also find that an arc of a circle or a line segment are stationary under (1.2) and (1.3). Our goal in this paper is to show a nonlinear stability result for stationary solutions to (1.2) and (1.3). A proof of such a result is difficult due to the area preserving property and due to the fact that highly nonlinear boundary conditions appear. We remark that for nonlinear boundary conditions satisfactory stability results are not available within the context of semigroup theory. We also remark that it is not possible to use methods based on maximum or comparison principles which have been used for mean curvature flow, see $[10,11]$.

For closed curves evolving by surface diffusion, Elliott and Garcke [7] showed a global existence result in the case that the initial curve is close to a circle. In addition, they proved nonlinear stability of circles under surface diffusion. Escher, Mayer, and Simonett [9] generalized the result in [7] to the higher-dimensional case. For evolving curves which come into contact with the outer boundary, Garcke, Ito, and Kohsaka [12] studied the linearized stability of stationary curves for (1.2) and (1.3). They derived a linearized stability criterion by extending the work for mean curvature flow of $[10,11,15]$ to motion by surface diffusion. For three evolving curves with a triple junction in the case that the outer boundary $\partial \Omega$ is a rectangle $[8,13]$ or a triangle [14], global existence results when the initial curve is a small perturbation of a certain stationary curve have been shown. Also nonlinear stability of this stationary curve can be shown.

Since the proof of nonlinear stability will heavily depend on the linear stability criterion derived in [12], we will now state it in detail. Let $\Gamma^{*}$ be a stationary curve parameterized by $X^{*}$ such that

$$
\Gamma^{*}=\left\{X^{*}(\sigma) \mid \sigma \in\left[l_{-}, l_{+}\right]\right\}
$$

where $\sigma$ is the arc-length parameter along $\Gamma^{*}$ and $X^{*}\left(l_{ \pm}\right) \in \partial \Omega$. Further, we denote by $\kappa^{*}$ the curvature of $\Gamma^{*}$ and by $h_{ \pm}^{*}$ the curvature of $\partial \Omega$ at $X^{*}\left(l_{ \pm}\right)$where we assume the sign convention that $h_{ \pm}^{*}$ is negative if $\Omega$ is convex. Then, the linearized stability criterion requires that

$$
I^{*}[w, w]=\int_{l_{-}}^{l_{+}}\left\{w_{\sigma}^{2}-\left(\kappa^{*}\right)^{2} w^{2}\right\} d \sigma+h_{+}^{*}\left(\left.w^{2}\right|_{\sigma=l_{+}}\right)+h_{-}^{*}\left(\left.w^{2}\right|_{\sigma=l_{-}}\right)
$$

is positive for all $w \in H^{1}\left(\Gamma^{*}\right)$ with mean value zero. In [12] this criterion was derived by studying the stability of the linearized problem. The same bilinear form also appears if one computes the second variation of the length functional taking boundary contacts into account, see e.g. Vogel [22]. We refer to Section 7 of [12] for several examples in which the linearized stability criterion has been applied. In the papers $[2,3]$ numerical results on the stability of stationary solutions for surface diffusion are presented. 
Our methods to obtain a nonlinear stability result are the following. First we introduce new curvilinear coordinates in order to derive an appropriate parameterization for which we can formulate (1.2) and (1.3) in a PDE setting. We then prove a local existence result, where the local existence time only depends on the $C^{2+\alpha}$-norm $(0<\alpha<1)$ of the initial curve. This is very helpful for a global existence result because we need a priori estimates only up to two spatial derivatives. In fact, by applying an energy method as in $[5,7,13,14]$ to a resulting evolution equation for the curvature, we can derive an a priori estimate of the $L^{2}$-norm of $\kappa_{s}$, which implies the boundedness of the $C^{2+\alpha}$-norm $(0<\alpha<1 / 2)$ of the solution for $t>0$. In the derivation of this a priori estimate, the linearized stability criterion developed in [12] is used. In addition, we need to understand the set of stationary solutions. We can use a result by Vogel [22] which guarantees that linearly stable stationary solutions are strict local minimizers of the length functional under an area constraint. We also show that in the neighborhood of the linearly stable stationary solution other stationary solutions can be parameterized by the enclosed area. This implies that the linearly stable stationary solution is isolated; a fact which will be important in order to study the long time behaviour of solutions.

This paper proceeds as follows. In Section 2, a parameterization established in [12] is employed for the geometric evolution equation (1.2) with boundary conditions (1.3). As a consequence, we obtain a nonlinear fourth order parabolic partial differential equation with nonlinear boundary conditions. We show a local existence result for this nonlinear parabolic problem. For the readers convenience we show an essential part of the proof of the local existence result in an Appendix. In Section 3, an evolution equation for the curvature is derived together with some geometric identities. The evolution equation for the curvature allows it to apply an energy method as in $[5,7,13,14]$. In Section 4 , we first derive a priori estimates for the length of $\Gamma_{t}$ and the $L^{2}$-norm of $\kappa_{s}$ when $\Gamma_{t}$ is close to a linearly stable stationary curve. These estimates imply the boundedness of the $C^{2+\alpha}$-norm $(0<\alpha<1 / 2)$ of the solution for $t>0$, so that the global existence result is proven when the initial curve is close to a linearly stable stationary curve. Finally, in Section 5, we show nonlinear stability of linearly stable stationary curves.

\section{Local existence and uniqueness}

In order to derive local existence and uniqueness for the geometric evolution equation (1.2) with the boundary conditions (1.3), we employ a parameterization which was established in [12]. For the readers convenience, we give a detailed derivation of the parameterization in the following.

Let $\Omega \subset \mathbb{R}^{2}$ be a domain such that

$$
\Omega=\left\{x \in \mathbb{R}^{2} \mid \psi(x)<0\right\}, \quad \partial \Omega=\left\{x \in \mathbb{R}^{2} \mid \psi(x)=0\right\}
$$

with a smooth function $\psi: \mathbb{R}^{2} \rightarrow \mathbb{R}$ fulfilling $\nabla \psi(x) \neq 0$ for $x$ with $\psi(x)=0$.

Also, let $\Gamma^{*}$ be a stationary curve under the flow (1.2) and (1.3), i.e. $\Gamma^{*}$ has constant curvature $\kappa^{*}$. We now introduce an arc-length parameterization of $\Gamma^{*}$ in the form

$$
\Gamma^{*}=\left\{\Phi^{*}(\sigma) \mid \sigma \in\left[l_{-}, l_{+}\right]\right\},
$$

where $\Phi^{*}$ is a mapping from $\left[l_{-}, l_{+}\right]$to $\mathbb{R}^{2}$ and $l_{+}-l_{-}$is the total length of $\Gamma^{*}$. Note that we can extend $\Gamma^{*}$ naturally either to the full circle when $\Gamma^{*}$ is an arc of a circle or to a 
straight line when $\Gamma^{*}$ is a line segment. We set

$$
\bar{l}:= \begin{cases}\pi /\left|\kappa^{*}\right|, & \text { if } \kappa^{*} \neq 0 \\ +\infty, & \text { if } \kappa^{*}=0\end{cases}
$$

i.e. $\bar{l}$ is the length of the extension of $\Gamma^{*}$ to a half circle if $\kappa_{*} \neq 0$. Without loss of generality, we can assume $\left[l_{-}, l_{+}\right] \subset(-\bar{l}, \bar{l})$. Define

$$
\left\{\begin{array}{l}
\xi_{+}(q):=\max \left\{\sigma \in(-\bar{l}, \bar{l}) \mid \Phi^{*}(\sigma)+q N^{*}(\sigma) \in \Omega\right\}, \\
\xi_{-}(q):=\min \left\{\sigma \in(-\bar{l}, \bar{l}) \mid \Phi^{*}(\sigma)+q N^{*}(\sigma) \in \Omega\right\}
\end{array}\right.
$$

where $N^{*}(\sigma)$ is a unit normal vector of $\Gamma^{*}$ at $\sigma$ and is obtained by rotating the unit tangent vector $T^{*}(\sigma)$ of $\Gamma^{*}$ by $\pi / 2$. Above, $q$ is a parameter with $q \in(-\bar{d}, \bar{d})$ for a small and given $\bar{d}>0$. It holds that $\psi\left(\Phi^{*}\left(\xi_{ \pm}(q)\right)+q N^{*}\left(\xi_{ \pm}(q)\right)\right)=0$ and $\xi_{ \pm}(0)=l_{ \pm}$. Using the implicit function theorem, we see that $\xi_{+}(q)$ and $\xi_{-}(q)$ are smooth. Let

$$
\Psi(\sigma, q):=\Phi^{*}(\xi(\sigma, q))+q N^{*}(\xi(\sigma, q))
$$

with

$$
\xi(\sigma, q):=\xi_{-}(q)+\frac{\sigma-l_{-}}{l_{+}-l_{-}}\left(\xi_{+}(q)-\xi_{-}(q)\right) .
$$

It is not difficult to check that $\xi\left(l_{ \pm}, q\right)=\xi_{ \pm}(q)$ and $\xi(\sigma, 0)=\sigma$.

In addition, one derives that $\Psi:\left(l_{-}, l_{+}\right) \times(-\bar{d}, \bar{d}) \rightarrow \Omega$ parametrizes the intersection $W$ of a tubular neighborhood around the extended $\Gamma^{*}$ with $\Omega$. We now consider functions $\rho:\left[l_{-}, l_{+}\right] \rightarrow(-\bar{d}, \bar{d})$ and obtain $\Psi(\sigma, \rho(\sigma)) \in W$ for $\sigma \in\left(l_{-}, l_{+}\right)$. Then we define $\Phi(\sigma):=\Psi(\sigma, \rho(\sigma))$ for $\sigma \in\left[l_{-}, l_{+}\right]$, which is a parameterization of a curve $\Gamma$. An evolving curve is now given by

$$
\Gamma_{t}:=\left\{\Phi(\sigma, t) \mid \sigma \in\left[l_{-}, l_{+}\right]\right\}
$$

with $\Phi(\sigma, t):=\Psi(\sigma, \rho(\sigma, t))$ for a function $\rho=\rho(\sigma, t)$. We note that $|\rho(\sigma, t)|<\bar{d}$ guarantees that $\Phi(\sigma, t)=\Psi(\sigma, \rho(\sigma, t)) \in W$ for $\sigma \in\left(l_{-}, l_{+}\right)$and $t>0$. We remark that $\rho \equiv 0$ corresponds to the stationary curve $\Gamma^{*}$.

Let us now express (1.2) and (1.3) with the help of parameterizations which have the form (2.1). For the arc-length parameter $s$ of $\Gamma_{t}$, we have

$$
\frac{d s}{d \sigma}=\left|\Phi_{\sigma}\right|=\sqrt{\left|\Psi_{\sigma}\right|^{2}+2\left(\Psi_{\sigma}, \Psi_{q}\right)_{\mathbb{R}^{2}} \rho_{\sigma}+\left|\Psi_{q}\right|^{2} \rho_{\sigma}^{2}}=: J(\rho) .
$$

By $|\cdot|$ and $(\cdot, \cdot)_{\mathbb{R}^{2}}$ we denote the norm and the inner product in $\mathbb{R}^{2}$, respectively. Then we find

$$
T=\frac{1}{J(\rho)} \Phi_{\sigma}, \quad N=\frac{1}{J(\rho)} R \Phi_{\sigma}
$$

where $T$ and $N$ are the unit tangent and unit normal to $\Gamma_{t}$, respectively, and $R$ is the rotation by the angle $\pi / 2$. The normal velocity $V$ of $\Gamma_{t}$ is given by

$$
V=\left(\Phi_{t}, N\right)_{\mathbb{R}^{2}}=\frac{1}{J(\rho)}\left(\Phi_{t}, R \Phi_{\sigma}\right)_{\mathbb{R}^{2}}=\frac{1}{J(\rho)}\left(\Psi_{q}, R \Psi_{\sigma}\right)_{\mathbb{R}^{2}} \rho_{t}
$$

Further, the Laplace-Beltrami operator $\Delta(\rho)$ on $\Gamma_{t}$ is given via $(2.2)$ as

$$
\Delta(\rho)=\partial_{s}^{2}=\frac{1}{J(\rho)} \partial_{\sigma}\left(\frac{1}{J(\rho)} \partial_{\sigma}\right)=\frac{1}{(J(\rho))^{2}} \partial_{\sigma}^{2}+\frac{1}{J(\rho)}\left(\partial_{\sigma} \frac{1}{J(\rho)}\right) \partial_{\sigma} .
$$


Then, the curvature $\kappa$ of $\Gamma_{t}$ can be derived by using $\Delta(\rho)$ as

$$
\begin{aligned}
\kappa(\rho)= & (\Delta(\rho) \Phi, N)_{\mathbb{R}^{2}}=\frac{1}{(J(\rho))^{3}}\left(\Phi_{\sigma \sigma}, R \Phi_{\sigma}\right)_{\mathbb{R}^{2}} \\
= & \frac{1}{(J(\rho))^{3}}\left[\left(\Psi_{q}, R \Psi_{\sigma}\right)_{\mathbb{R}^{2}} \rho_{\sigma \sigma}+\left\{2\left(\Psi_{\sigma q}, R \Psi_{\sigma}\right)_{\mathbb{R}^{2}}+\left(\Psi_{\sigma \sigma}, R \Psi_{q}\right)_{\mathbb{R}^{2}}\right\} \rho_{\sigma}\right. \\
& +\left\{\left(\Psi_{q q}, R \Psi_{\sigma}\right)_{\mathbb{R}^{2}}+2\left(\Psi_{\sigma q}, R \Psi_{q}\right)_{\mathbb{R}^{2}}+\left(\Psi_{q q}, R \Psi_{q}\right)_{\mathbb{R}^{2}} \rho_{\sigma}\right\} \rho_{\sigma}^{2} \\
& \left.+\left(\Psi_{\sigma \sigma}, R \Psi_{\sigma}\right)_{\mathbb{R}^{2}}\right]
\end{aligned}
$$

Furthermore, we note that the Neumann boundary condition $\left(\Phi_{\sigma}, T_{\partial \Omega}\right)_{\mathbb{R}^{2}}=0$ on $\partial \Omega$ is equivalent to the condition $\left(R \Phi_{\sigma}, \nabla \psi(\Phi)\right)_{\mathbb{R}^{2}}=0$ on $\partial \Omega$. Then we compute that the parameterization of the Neumann boundary condition is

$$
\left(R \Psi_{\sigma}+R \Psi_{q} \rho_{\sigma}, \nabla \psi(\Psi)\right)_{\mathbb{R}^{2}}=0 \text { at } \sigma=l_{ \pm}
$$

As a consequence, we conclude that the problem (1.2) and (1.3) is represented by

$$
\begin{cases}\rho_{t}=-L(\rho) \Delta(\rho) \kappa(\rho) & \text { for } \sigma \in\left(l_{-}, l_{+}\right), t>0 \\ \left(R \Psi_{\sigma}+R \Psi_{q} \rho_{\sigma}, \nabla \psi(\Psi)\right)_{\mathbb{R}^{2}}=0 & \text { at } \sigma=l_{ \pm}, \\ \partial_{\sigma} \kappa(\rho)=0 & \text { at } \sigma=l_{ \pm} .\end{cases}
$$

Here $L(\rho):=J(\rho) /\left(\Psi_{q}, R \Psi_{\sigma}\right)_{\mathbb{R}^{2}} ; \Delta(\rho)$ and $\kappa(\rho)$ are given by (2.3) and (2.4), respectively.

Let $\mathcal{I}=\left[l_{-}, l_{+}\right]$and $\mathcal{Q}_{t_{0}, t_{1}}=\mathcal{I} \times\left(t_{0}, t_{1}\right]$ for $0 \leq t_{0}<t_{1}<\infty$. For $0<\alpha<1$, we define the function space

$$
\mathcal{Y}\left(\overline{\mathcal{Q}_{t_{0}, t_{1}}}\right)=\left\{\rho \in C^{2+\alpha, 0}\left(\overline{\mathcal{Q}_{t_{0}, t_{1}}}\right) \cap C^{4+\alpha, 1}\left(\mathcal{Q}_{t_{0}, t_{1}}\right) \mid\|\rho\|_{\mathcal{Y}\left(\overline{\mathcal{Q}_{t_{0}, t_{1}}}\right)}<\infty\right\}
$$

with the norm

$$
\begin{aligned}
\|\rho\|_{\mathcal{Y}\left(\overline{\mathcal{Q}_{0}, t_{1}}\right)}= & \sup _{t_{0} \leq t \leq t_{1}}\|\rho(\cdot, t)\|_{C^{2+\alpha}(\mathcal{I})}+\sup _{t_{0}<t \leq t_{1}}\left(t-t_{0}\right)^{1 / 2}\left\|\partial_{\sigma}^{4} \rho(\cdot, t)\right\|_{C^{\alpha}(\mathcal{I})} \\
& +\sup _{t_{0}<t \leq t_{1}}\left(t-t_{0}\right)^{1 / 2}\left\|\rho_{t}(\cdot, t)\right\|_{C^{\alpha}(\mathcal{I})},
\end{aligned}
$$

where $\overline{\mathcal{Q}_{t_{0}, t_{1}}}$ is the closure of $\mathcal{Q}_{t_{0}, t_{1}}$.

Now we are ready to state a local existence theorem.

Theorem 2.1 (Local existence) Let $\alpha \in(0,1)$ and let us assume that $\rho_{0} \in C^{2+\alpha}(\mathcal{I})$ with $\left\|\rho_{0}\right\|_{C^{0}(\mathcal{I})}<\bar{d}$ fulfills

$$
\left(R \Psi_{\sigma}+R \Psi_{q} \rho_{\sigma}, \nabla \psi(\Psi)\right)_{\mathbb{R}^{2}}=0 \text { at } \sigma=l_{ \pm} .
$$

Then there exists a $T_{0}=T_{0}\left(1 /\left\|\rho_{0}\right\|_{C^{2+\alpha}(\mathcal{I})}\right)>0$ such that the problem (2.5) with $\rho(\cdot, 0)=$ $\rho_{0}$ has a unique solution in $\mathcal{Y}\left(\overline{\mathcal{Q}_{0, T_{0}}}\right)$.

This theorem is proved by applying similar arguments as in [13]. Since we have to take care of the boundary conditions in a different way, we will sketch the proof in the Appendix.

Remark 2.2 By using a bootstrapping argument as in [13, Theorem 3.6, Remark 3.7], it can be shown that the solution $\rho$ established in Theorem 2.1 is smooth for $t \in\left(0, T_{0}\right]$. 


\section{An evolution equation for curvature}

In order to show nonlinear stability of solutions for which the linearized stability criterion of [12] is fulfilled, we apply an energy method similar to the one used in $[5,7,13,14]$. For this approach it is important to derive an evolution equation for the curvature. Such an equation will allow it to derive a priori estimates using the linearized stability criterion.

For the above mentioned purpose, we employ a parameterization of the evolving curve $\Gamma_{t}$ by arc-length contrary to the one stated in Section 2 . Let $X$ be a smooth mapping so that $X(\cdot, t)$ is an arc-length parameterization of $\Gamma_{t}$, i.e.

$$
\Gamma_{t}:=\left\{X(s, t) \mid s \in\left[r_{-}(t), r_{+}(t)\right]\right\}
$$

for any $t>0$, where $r_{+}$and $r_{-}$are smooth in $t$. In particular, $X\left(r_{ \pm}(t), t\right) \in \partial \Omega$ and $r_{+}(t)-r_{-}(t)=L\left[\Gamma_{t}\right]$, where $L\left[\Gamma_{t}\right]$ denotes the total length of $\Gamma_{t}$. Let $N(=N(s, t))$ be the unit normal vector of $\Gamma_{t}$, which is represented as

$$
N(s, t)=\left(\begin{array}{c}
\cos \theta(s, t) \\
\sin \theta(s, t)
\end{array}\right)
$$

Also, let $T(=T(s, t))$ and $\kappa(=\kappa(s, t))$ be the unit tangent vector of $\Gamma_{t}$ and the curvature of $\Gamma_{t}$, respectively. Note that the unit tangent vector $T$ is obtained by rotating the unit normal vector $N$ by $-\pi / 2$. Then, using $\theta_{s}=\kappa$, we have

$$
\left\{\begin{array}{l}
N_{s}=-\kappa T, \quad T_{s}=\kappa N \\
N_{t}=-\theta_{t} T, \quad T_{t}=\theta_{t} N
\end{array}\right.
$$

In addition, set

$$
V:=\left(X_{t}, N\right)_{\mathbb{R}^{2}}, \quad v:=\left(X_{t}, T\right)_{\mathbb{R}^{2}} .
$$

Note that $V$ and $v$ are the normal velocity and the tangent velocity of $X$, respectively. Then it follows that

$$
X_{t}=V N+v T \text {. }
$$

Differentiating (3.2) with respect to $s$ and using (3.1), we have

$$
\begin{aligned}
X_{t s} & =V_{s} N+V N_{s}+v_{s} T+v T_{s} \\
& =\left(V_{s}+\kappa v\right) N+\left(-\kappa V+v_{s}\right) T .
\end{aligned}
$$

This implies the following lemma.

Lemma 3.1 Let $X$ be a smooth arc-length parameterization as above. Then

$$
\theta_{t}=V_{s}+\kappa v, \quad v_{s}=\kappa V .
$$

Proof. Since $X_{t s}=X_{s t}$ and $X_{s}=T$, it follows from (3.1) that

$$
\theta_{t} N=\left(V_{s}+\kappa v\right) N+\left(-\kappa V+v_{s}\right) T \text {. }
$$

Thus we obtain the desired results.

By Lemma 3.1, we have the following formula for the time-derivative of curvature. 
Lemma 3.2 Let $X$ be a smooth arc-length parameterization as above. Then

$$
\kappa_{t}=V_{s s}+\kappa^{2} V+\kappa_{s} v .
$$

Proof. By $\theta_{s}=\kappa$ and Lemma 3.1, we derive

$$
\kappa_{t}=\theta_{s t}=\theta_{t s}=\left(V_{s}+\kappa v\right)_{s}=V_{s s}+\kappa v_{s}+\kappa_{s} v=V_{s s}+\kappa^{2} V+\kappa_{s} v .
$$

This completes the proof.

By the assumption that $\Gamma_{t}$ touches $\partial \Omega$ with the angle $\pi / 2$, we have

$$
\psi\left(X\left(r_{ \pm}(t), t\right)\right)=0, \quad(\nabla \psi(X), N)_{\mathbb{R}^{2}}=0 \quad \text { at } s=r_{ \pm}(t) .
$$

Then we derive the following lemma.

Lemma 3.3 Let $X$ be a smooth arc-length parameterization as above. Then

$$
v\left(r_{ \pm}(t), t\right)+r_{ \pm}^{\prime}(t)=0
$$

Proof. Differentiating $\psi\left(X\left(r_{ \pm}(t), t\right)\right)=0$ with respect to $t$ and using $(\nabla \psi(X), N)_{\mathbb{R}^{2}}=0$ at $s=r_{ \pm}(t)$, we have at $s=r_{ \pm}(t)$

$$
\begin{aligned}
0 & =\left(\nabla \psi(X), X_{s} r_{ \pm}^{\prime}+X_{t}\right)_{\mathbb{R}^{2}}=\left(\nabla \psi(X), X_{s} r_{ \pm}^{\prime}+V N+v T\right)_{\mathbb{R}^{2}} \\
& =\left(v+r_{ \pm}^{\prime}\right)(\nabla \psi(X), T)_{\mathbb{R}^{2}}= \pm\left(v+r_{ \pm}^{\prime}\right)|\nabla \psi(X)| .
\end{aligned}
$$

The last identity is derived with the help of $T= \pm \nabla \psi(X) /|\nabla \psi(X)|$ at $s=r_{ \pm}(t)$. Since $|\nabla \psi(X)| \neq 0$, we obtain the desired result.

Now we can present an evolution equation for the curvature.

Proposition 3.4 (Evolution equation for the curvature) Let evolving curves $\Gamma=$ $\left\{\Gamma_{t}\right\}_{t \geq 0}$ be lying in $\Omega$ with $\partial \Gamma \subset \partial \Omega$. Then, a smooth solution of

$$
V=-\kappa_{s s} \quad \text { on } \quad \Gamma_{t}
$$

with the boundary conditions

$$
\left\{\begin{array}{lll}
\varangle\left(\Gamma_{t}, \partial \Omega\right)=\pi / 2 & \text { at } & \Gamma_{t} \cap \partial \Omega, \\
\kappa_{s}=0 & \text { at } & \Gamma_{t} \cap \partial \Omega
\end{array}\right.
$$

fulfills for $t>0$

$$
\kappa_{t}=-\kappa_{s s s}-\kappa^{2} \kappa_{s s}+\kappa_{s} v \quad \text { on } \quad \Gamma_{t}
$$

and

$$
\left\{\begin{array}{lll}
\kappa_{s}=0 & \text { at } & \Gamma_{t} \cap \partial \Omega \\
\left(\partial_{s} \pm h_{ \pm}\right) \kappa_{s s}=0 & \text { at } & \Gamma_{t} \cap \partial \Omega
\end{array}\right.
$$

Here $h_{ \pm}$is the curvature of $\partial \Omega$ at the points $X\left(r_{ \pm}(t), t\right) \in \Gamma_{t} \cap \partial \Omega$ with the sign convention that $h_{ \pm} \leq 0$ if $\Omega$ is convex 
Proof. We immediately obtain (3.5) from (3.3) and Lemma 3.2. Next we show (3.6). Differentiating $(\nabla \psi(X), N)_{\mathbb{R}^{2}}=0$ at $s=r_{ \pm}(t)$ with respect to $t$ and using (3.1), (3.2), Lemma 3.1, and Lemma 3.3, we have at $s=r_{ \pm}(t)$

$$
\begin{aligned}
0= & \left(\left[D^{2} \psi(X)\right]\left(X_{s} r_{ \pm}^{\prime}+X_{t}\right), N\right)_{\mathbb{R}^{2}}+\left(\nabla \psi(X), N_{s} r_{ \pm}^{\prime}+N_{t}\right)_{\mathbb{R}^{2}} \\
= & \left(v+r_{ \pm}^{\prime}\right)\left(\left[D^{2} \psi(X)\right] T, N\right)_{\mathbb{R}^{2}}+V\left(\left[D^{2} \psi(X)\right] N, N\right)_{\mathbb{R}^{2}} \\
& -\kappa r_{ \pm}^{\prime}(\nabla \psi(X), T)_{\mathbb{R}^{2}}-\theta_{t}(\nabla \psi(X), T)_{\mathbb{R}^{2}} \\
= & V\left(\left[D^{2} \psi(X)\right] T_{\partial \Omega}(X), T_{\partial \Omega}(X)\right)_{\mathbb{R}^{2}}-V_{s}(\nabla \psi(X), T)_{\mathbb{R}^{2}} \\
& -\kappa\left(v+r_{ \pm}^{\prime}\right)(\nabla \psi(X), T)_{\mathbb{R}^{2}} \\
= & V\left(\left[D^{2} \psi(X)\right] T_{\partial \Omega}(X), T_{\partial \Omega}(X)\right)_{\mathbb{R}^{2}} \mp V_{s}|\nabla \psi(X)| .
\end{aligned}
$$

Here $D^{2} \psi$ is the Hessian matrix of $\psi$. Then we observe

$$
\kappa_{\partial \Omega}(X)=-\frac{1}{|\nabla \psi(X)|}\left(\left[D^{2} \psi(X)\right] T_{\partial \Omega}(X), T_{\partial \Omega}(X)\right)_{\mathbb{R}^{2}}
$$

so that

$$
V_{s} \pm h_{ \pm} V=0 \quad \text { at } s=r_{ \pm}(t)
$$

where $h_{ \pm}$are given by $h_{ \pm}:=\kappa_{\partial \Omega}\left(X\left(r_{ \pm}(t), t\right)\right)$. This completes the proof.

\section{A priori estimates and global existence}

We now derive basic evolution formulas for length and $\int_{\Gamma_{t}} \kappa_{s}^{2} d s$.

Lemma 4.1 A smooth solution of (3.3)-(3.4) fulfills

(i) $\frac{d}{d t} L\left[\Gamma_{t}\right]=-\int_{\Gamma_{t}} \kappa_{s}^{2} d s$,

(ii) $\frac{d}{d t} \int_{\Gamma_{t}} \kappa_{s}^{2} d s=-2\left\{\int_{\Gamma_{t}} V_{s}^{2} d s-\int_{\Gamma_{t}} \kappa^{2} V^{2} d s+h_{+}\left(\left.V^{2}\right|_{s=r_{+}(t)}\right)+h_{-}\left(\left.V^{2}\right|_{s=r_{-}(t)}\right)\right\}$

$$
+\int_{\Gamma_{t}} \kappa_{s}^{2} \kappa V d s
$$

where $h_{ \pm}$is evaluated at $X\left(r_{ \pm}(t), t\right)$.

Proof. Recalling $L\left[\Gamma_{t}\right]=r_{+}(t)-r_{-}(t)$ and using Lemma 3.1 and Lemma 3.3, we have

$$
\begin{aligned}
\frac{d}{d t} L\left[\Gamma_{t}\right] & =r_{+}^{\prime}(t)-r_{-}^{\prime}(t)=-v\left(r_{+}(t), t\right)+v\left(r_{-}(t), t\right)=-\int_{\Gamma_{t}} v_{s} d s \\
& =-\int_{\Gamma_{t}} \kappa V d s=\int_{\Gamma_{t}} \kappa \kappa_{s s} d s=-\int_{\Gamma_{t}} \kappa_{s}^{2} d s .
\end{aligned}
$$

The last term is derived using integration by parts and $\kappa_{s}=0$ at $\Gamma_{t} \cap \partial \Omega$.

In order to prove (ii), we compute

$$
\int_{\Gamma_{t}} \kappa_{s}\left(\kappa_{t}\right)_{s} d s=\int_{\Gamma_{t}} \kappa_{s}\left(-\kappa_{s s s s}-\kappa^{2} \kappa_{s s}+\kappa_{s} v\right)_{s} d s
$$


Since $\kappa_{t s}=\kappa_{s t}$ and $\kappa_{s}=0$ at $\Gamma_{t} \cap \partial \Omega$, we have

$$
(\text { L.H.S. of }(4.1))=\int_{\Gamma_{t}} \kappa_{s} \kappa_{s t} d s=\frac{1}{2} \int_{\Gamma_{t}}\left(\kappa_{s}^{2}\right)_{t} d s=\frac{1}{2} \frac{d}{d t} \int_{\Gamma_{t}} \kappa_{s}^{2} d s .
$$

On the other hand, by means of integration by parts and using (3.6), we derive

$$
\begin{aligned}
\text { (R.H.S. of (4.1)) }= & -\int_{\Gamma_{t}} \kappa_{s s}\left(-\kappa_{s s s s}-\kappa^{2} \kappa_{s s}+\kappa_{s} v\right) d s \\
= & \int_{\Gamma_{t}} \kappa_{s s} \kappa_{s s s s} d s+\int_{\Gamma_{t}} \kappa^{2} \kappa_{s s}^{2} d s-\int_{\Gamma_{t}} \kappa_{s s} \kappa_{s} v d s \\
= & -h_{+}\left(\left.\kappa_{s s}^{2}\right|_{s=r_{+}(t)}\right)-h_{-}\left(\left.\kappa_{s s}^{2}\right|_{s=r_{-}(t)}\right)-\int_{\Gamma_{t}} \kappa_{s s s}^{2} d s \\
& +\int_{\Gamma_{t}} \kappa^{2} \kappa_{s s}^{2} d s+\frac{1}{2} \int_{\Gamma_{t}} \kappa_{s}^{2} v_{s} d s .
\end{aligned}
$$

Thus it follows from $V=-\kappa_{s s}$ and $v_{s}=\kappa V$ that

$$
\begin{aligned}
\frac{1}{2} \frac{d}{d t} \int_{\Gamma_{t}} \kappa_{s}^{2} d s= & -\left\{\int_{\Gamma_{t}} V_{s}^{2} d s-\int_{\Gamma_{t}} \kappa^{2} V^{2} d s+h_{+}\left(\left.V^{2}\right|_{s=r_{+}(t)}\right)+h_{-}\left(\left.V^{2}\right|_{s=r_{-}(t)}\right)\right\} \\
& +\frac{1}{2} \int_{\Gamma_{t}} \kappa_{s}^{2} \kappa V d s .
\end{aligned}
$$

This completes the proof.

Let us define the bilinear form $I$ as

$$
I[w, w]=\int_{r_{-}}^{r_{+}}\left(w_{s}^{2}-\kappa_{a v}^{2} w^{2}\right) d s+h_{+}\left(\left.w^{2}\right|_{s=r_{+}}\right)+h_{-}\left(\left.w^{2}\right|_{s=r_{-}}\right)
$$

for $w \in H^{1}\left(\Gamma_{t}\right)$ with

$$
\int_{r_{-}}^{r_{+}} w d s=0
$$

Here $s$ is the arc-length parameter along $\Gamma_{t}$, which belongs to the interval $\left[r_{-}, r_{+}\right]$with $L\left[\Gamma_{t}\right]=r_{+}-r_{-} ; h_{ \pm}$is the curvature of $\partial \Omega$ at $\Gamma_{t} \cap \partial \Omega$; and $\kappa_{a v}$ is the averaged curvature of $\Gamma_{t}$ defined as

$$
\kappa_{a v}=\frac{1}{L\left[\Gamma_{t}\right]} \int_{r_{-}}^{r_{+}} \kappa d s .
$$

Since $V=-\kappa_{s s}$ and $\kappa_{s}=0$ at $\Gamma_{t} \cap \partial \Omega$, it holds that

$$
\int_{\Gamma_{t}} V d s=0
$$

Then, we can rewrite Lemma 4.1 (ii) as

$$
\frac{d}{d t} \int_{\Gamma_{t}} \kappa_{s}^{2} d s+2 I[V, V]=-2 \int_{\Gamma_{t}}\left(\kappa_{a v}^{2}-\kappa^{2}\right) V^{2} d s+\int_{\Gamma_{t}} \kappa_{s}^{2} \kappa V d s
$$

The following lemmas will be crucial in order to derive an a priori estimate. 
Lemma 4.2 A smooth solution of (3.3)-(3.4) fulfills

(i) $\left|\int_{\Gamma_{t}} \kappa_{s}^{2} \kappa \kappa_{s s} d s\right| \leq \frac{1}{3} L\left[\Gamma_{t}\right]\left\|\kappa_{s}\right\|_{L^{2}\left(\Gamma_{t}\right)}^{2}\left\|\kappa_{s s}\right\|_{L^{2}\left(\Gamma_{t}\right)}^{2}$.

(ii) $\left\|\kappa-\kappa_{a v}\right\|_{C^{0}\left(\Gamma_{t}\right)} \leq L\left[\Gamma_{t}\right]^{1 / 2}\left\|\kappa_{s}\right\|_{L^{2}\left(\Gamma_{t}\right)}$.

Proof. We first prove (i). Since $\kappa_{s}=0$ at $\Gamma_{t} \cap \partial \Omega$, we get

$$
\int_{\Gamma_{t}} \kappa_{s}^{2} \kappa \kappa_{s s} d s=-\frac{1}{3} \int_{\Gamma_{t}} \kappa_{s}^{4} d s
$$

Then it follows that

$$
\begin{aligned}
\left|\int_{\Gamma_{t}} \kappa_{s}^{4} d s\right| & \leq\left\|\kappa_{s}\right\|_{L^{2}\left(\Gamma_{t}\right)}^{2}\left\|\kappa_{s}\right\|_{L^{\infty}\left(\Gamma_{t}\right)}^{2} \\
& \leq L\left[\Gamma_{t}\right]\left\|\kappa_{s}\right\|_{L^{2}\left(\Gamma_{t}\right)}^{2}\left\|\kappa_{s s}\right\|_{L^{2}\left(\Gamma_{t}\right)}^{2} .
\end{aligned}
$$

The last term is derived by using a Poincaré inequality since $\kappa_{s}=0$ at $\Gamma_{t} \cap \partial \Omega$.

Next we prove (ii). Since

$$
\int_{\Gamma_{t}}\left(\kappa-\kappa_{a v}\right) d s=0
$$

for each $t>0$, there is a $r_{0}\left(=r_{0}(t)\right) \in\left(r_{-}(t), r_{+}(t)\right)$ such that $\kappa\left(r_{0}, t\right)-\kappa_{a v}(t)=0$. This implies that

$$
\left|\kappa(s, \cdot)-\kappa_{a v}\right|=\left|\int_{r_{0}}^{s}\left(\kappa-\kappa_{a v}\right)_{s} d s\right|=\left|\int_{r_{0}}^{s} \kappa_{s} d s\right| \leq \int_{\Gamma_{t}}\left|\kappa_{s}\right| d s \leq L\left[\Gamma_{t}\right]^{1 / 2}\left\|\kappa_{s}\right\|_{L^{2}\left(\Gamma_{t}\right)} .
$$

Thus we have the desired result.

We remind the reader that for functions $w_{1}, w_{2}$ with mean values zero we can define the $H^{-1}$-inner product via

$$
\left(w_{1}, w_{2}\right)_{-1}=\int_{l_{-}}^{l_{+}} u_{1, \sigma} u_{2, \sigma} d \sigma
$$

where $u_{i}$ is the solution of $-u_{i, \sigma \sigma}=w_{i}$ in $\left(l_{-}, l_{+}\right)$and $u_{i, \sigma}=0$ at $\sigma=l_{ \pm}$. According to [12], the bilinear form $I^{*}$ as stated in the introduction, see (1.4), is positive provided that the maximal eigenvalue $\lambda$ for the linearized problem to (1.2) and (1.3) is negative. In [12] it was shown that $I[w, w] \geq(-\lambda)(w, w)_{-1}$ for all $w$ with mean value zero. We now want to derive a perturbation of this result. Let us denote $L=L[\Gamma]$ and $L^{*}=L\left[\Gamma^{*}\right]\left(=l_{+}-l_{-}\right)$. Then we have the following lemma, which implies a lower bound for $I$ when the parameters $\kappa_{a v}, h_{ \pm}$, and $L$ are close to $\kappa^{*}, h_{ \pm}^{*}$, and $L^{*}$, respectively.

Lemma 4.3 (i) Let $\lambda$ be the maximal eigenvalue of the linearized problem. For $\varepsilon>0$ there exists $\delta>0$ such that

$$
I[w, w]>(-\lambda-\varepsilon)(w, w)_{-1}
$$

for $w \in H^{1}(\Gamma)$ with mean value zero provided that

$$
\left|\kappa_{a v}-\kappa^{*}\right|<\delta, \quad\left|h_{ \pm}-h_{ \pm}^{*}\right|<\delta, \quad\left|L-L^{*}\right|<\delta .
$$


(ii) There exists $\mu>0$ such that

$$
\mu\left\|w_{s}\right\|_{L^{2}(\Gamma)}^{2} \leq I[w, w]+(w, w)_{-1}
$$

for $w \in H^{1}(\Gamma)$ with mean value zero.

Proof. The largest eigenvalue $\lambda$ corresponding to the bilinear form $I$ depends continuously on $L, \kappa_{a v}, h_{ \pm}$. In the case that $L=L^{*}, \kappa_{a v}=\kappa^{*}, h_{ \pm}=h_{ \pm}^{*}$ we obtain (i) with $\varepsilon=0$ and hence (i) follows from a straight forward perturbation argument, compare [12] for similar arguments. Arguing as in the proof of Lemma 5.3 in [12], we obtain (ii).

It is significant to obtain a positive lower bound of $L[\Gamma]$ in terms of $\rho$. The following lemma implies that $L^{*}$ is a local minimum of $L[\Gamma]$ provided that $I^{*}$ is positive.

Lemma 4.4 Let $\Gamma^{*}$ be a stationary curve such that the bilinear form $I^{*}$ is positive and let $\rho \in C^{1}(\mathcal{I})$ be a function describing a curve $\Gamma$ close to $\Gamma^{*}$ as in Section 2. Assume that a curve $\Gamma$ encloses the same area as $\Gamma^{*}$. Then there exist constants $\bar{c}, \gamma^{*}>0$ such that

$$
L[\Gamma] \geq L^{*}+\bar{c}\|\rho\|_{H^{1}(\mathcal{I})}^{2}
$$

if $\|\rho\|_{C^{1}(\mathcal{I})}<\gamma^{*}$.

Proof. This follows as in the proof of Theorem 2.1 of Vogel [22] (see (2.14) and the inequality after (2.19) in [22]).

By virtue of Lemma 4.4, we have an a priori estimate of $L\left[\Gamma_{t}\right]$ and can derive useful estimates concerning $\kappa_{a v}$ and $h_{ \pm}$.

Lemma 4.5 Let the assumptions of Lemma 4.4 hold for a stationary curve $\Gamma^{*}$ and all curves $\Gamma_{t}, t \in[0, T]$, described by $\rho(t) \in C^{1}(\mathcal{I})$ for the parameterization in Section 2. Assume in particular that $\|\rho(t)\|_{C^{1}(\mathcal{I})}<\gamma^{*}$ for $t \in[0, T]$ where $\gamma^{*}$ is as in Lemma 4.4. We then obtain:

(i) $L\left[\Gamma_{0}\right] \geq L\left[\Gamma_{t}\right] \geq L^{*}$ for all $t \in[0, T]$.

(ii) There exist $K_{1}, K_{2}>0$ such that for $t \in[0, T]$

$$
\left|\kappa_{a v}(t)-\kappa^{*}\right| \leq K_{1}\left|L\left[\Gamma_{t}\right]-L^{*}\right|, \quad\left|h_{ \pm}(t)-h_{ \pm}^{*}\right| \leq K_{2}\left|L\left[\Gamma_{t}\right]-L^{*}\right| .
$$

Proof. (i) follows from Lemma 4.1(i) and Lemma 4.4. To prove (ii), we compute

$$
\kappa_{a v}=\frac{1}{L\left[\Gamma_{t}\right]} \int_{\Gamma_{t}} \kappa d s=\frac{1}{L\left[\Gamma_{t}\right]} \int_{\Gamma_{t}} \theta_{s} d s=\frac{1}{L\left[\Gamma_{t}\right]}\left(\theta_{+}-\theta_{-}\right)
$$

A similar computation gives

$$
\kappa^{*}=\frac{1}{L^{*}}\left(\theta_{+}^{*}-\theta_{-}^{*}\right) .
$$


Then we have

$$
\begin{aligned}
\left|\kappa_{a v}-\kappa^{*}\right| & =\left|\frac{1}{L\left[\Gamma_{t}\right]}\left(\theta_{+}-\theta_{-}\right)-\frac{1}{L^{*}}\left(\theta_{+}^{*}-\theta_{-}^{*}\right)\right| \\
& =\frac{1}{L\left[\Gamma_{t}\right] L^{*}}\left|L^{*}\left(\theta_{+}-\theta_{-}\right)-L\left[\Gamma_{t}\right]\left(\theta_{+}^{*}-\theta_{-}^{*}\right)\right| \\
& \leq\left(\frac{1}{L^{*}}\right)^{2}\left\{\left|L^{*}\left(\theta_{+}-\theta_{-}-\left(\theta_{+}^{*}-\theta_{-}^{*}\right)\right)\right|+\left|L^{*}-L\left[\Gamma_{t}\right]\right|\left|\theta_{+}^{*}-\theta_{-}^{*}\right|\right\} .
\end{aligned}
$$

By means of the mean value theorem, the smoothness of $\partial \Omega$, and the $\pi / 2$ angle condition, we see that the quantity $\left|\theta_{+}-\theta_{+}^{*}\right|+\left|\theta_{-}^{*}-\theta_{-}\right|$is estimated by $\|\rho\|_{C^{0}(\mathcal{I})}$. Using Lemma 4.4 and an embedding result, we obtain the first inequality in (ii).

Recall that $\kappa_{\partial \Omega}(X)$ is represented by

$$
\kappa_{\partial \Omega}(X)=-\frac{1}{|\nabla \psi(X)|}\left(\left[D^{2} \psi(X)\right] T_{\partial \Omega}(X), T_{\partial \Omega}(X)\right)_{\mathbb{R}^{2}}
$$

Since this expression does not depend on derivatives of $\rho$, the mean value theorem implies that the quantity $\left|h_{ \pm}-h_{ \pm}^{*}\right|$ is estimated by $\|\rho\|_{C^{0}(\mathcal{I})}$. Using Lemma 4.4 and an embedding result, we derive the second inequality in (ii) .

Using Lemma 4.3, we obtain the existence of constants $\delta^{*}>0$ and $\mu^{*}>0$ such that

$$
I[w, w]>-\frac{\lambda}{2}(w, w)_{-1}+\mu^{*}\left\|w_{s}\right\|_{L^{2}\left(\Gamma_{t}\right)}^{2}
$$

for $w \in H^{1}\left(\Gamma_{t}\right)$ with mean value zero provided that

$$
\left|\kappa_{a v}(t)-\kappa^{*}\right|<\delta^{*}, \quad\left|h_{ \pm}(t)-h_{ \pm}^{*}\right|<\delta^{*}, \quad\left|L\left[\Gamma_{t}\right]-L^{*}\right|<\delta^{*} .
$$

We are now in a position to derive a priori estimates for solutions of (2.5) if the solution is close to $\Gamma^{*}$.

Proposition 4.6 Let the assumptions of Lemma 4.4 hold for a stationary curve $\Gamma^{*}$ and a curve $\Gamma_{t}$ described by $\rho(t) \in C^{1}(\mathcal{I})$ for the parameterization in Section 2. Assume that for $t \in(0, T]$

$$
\|\rho(t)\|_{C^{1}(\mathcal{I})}<\gamma^{*} \quad \text { and } \quad\left|L\left[\Gamma_{t}\right]-L^{*}\right| \leq \frac{\delta^{*}}{1+K_{1}+K_{2}}\left(=: \delta_{1}^{*}\right),
$$

where $\gamma^{*}$ is as in Lemma 4.4, $K_{1}$ and $K_{2}$ are as in Lemma 4.5 and $\delta^{*}$ is as in (4.5). Then there is a constant $\delta_{1}>0$ such that if $\left\|\kappa_{s}(t)\right\|_{L^{2}\left(\Gamma_{t}\right)}^{2}<\delta_{1}$ for $t \in(0, T]$, it holds

$$
\left\|\kappa_{s}(t)\right\|_{L^{2}\left(\Gamma_{t}\right)}^{2}+\mu^{*} \int_{t_{0}}^{t}\left\|V_{s}(\tau)\right\|_{L^{2}\left(\Gamma_{t}\right)}^{2} d \tau \leq\left\|\kappa_{s}\left(t_{0}\right)\right\|_{L^{2}\left(\Gamma_{t}\right)}^{2}
$$

for $t \in\left[t_{0}, T\right]$ with $t_{0}>0$ where $\mu^{*}$ is as in (4.4). 
Proof. By (4.3), we have

$$
\begin{aligned}
& \frac{d}{d t}\left\|\kappa_{s}\right\|_{L^{2}\left(\Gamma_{t}\right)}^{2}+2 I[V, V] \\
& =-2 \int_{\Gamma_{t}}\left(\kappa_{a v}^{2}-\kappa^{2}\right) V^{2} d s+\int_{\Gamma_{t}} \kappa_{s}^{2} \kappa V d s \\
& =2 \int_{\Gamma_{t}}\left(\kappa-\kappa_{a v}\right)^{2} V^{2} d s+4 \kappa_{a v} \int_{\Gamma_{t}}\left(\kappa-\kappa_{a v}\right) V^{2} d s+\int_{\Gamma_{t}} \kappa_{s}^{2} \kappa V d s .
\end{aligned}
$$

By virtue of (4.6) and Lemma 4.5(ii), we also see that $\kappa_{a v}(t), h_{ \pm}(t)$, and $L\left[\Gamma_{t}\right]$ satisfy (4.5). Then it follows from Lemma 4.2, Lemma 4.5(i), and (4.4) that there exist $C_{1}, C_{2}>0$ such that

$$
\begin{aligned}
& \frac{d}{d t}\left\|\kappa_{s}\right\|_{L^{2}\left(\Gamma_{t}\right)}^{2}+(-\lambda)(V, V)_{-1}+2 \mu^{*}\left\|V_{s}\right\|_{L^{2}\left(\Gamma_{t}\right)}^{2} \\
& \leq C_{1}\|V\|_{L^{2}\left(\Gamma_{t}\right)}^{2}\left\|\kappa_{s}\right\|_{L^{2}\left(\Gamma_{t}\right)}^{2}+C_{2}\left(\delta^{*}+\left|\kappa^{*}\right|\right)\|V\|_{L^{2}\left(\Gamma_{t}\right)}^{2}\left\|\kappa_{s}\right\|_{L^{2}\left(\Gamma_{t}\right)} .
\end{aligned}
$$

Since $\|V\|_{L^{\infty}\left(\Gamma_{t}\right)} \leq C\left\|V_{s}\right\|_{L^{2}\left(\Gamma_{t}\right)}$ by virtue of (4.2), we derive $\|V\|_{L^{2}\left(\Gamma_{t}\right)} \leq \widetilde{C}\left\|V_{s}\right\|_{L^{2}\left(\Gamma_{t}\right)}$. By means of this fact and $(-\lambda)(V, V)_{-1} \geq 0$, we are led to

$$
\frac{d}{d t}\left\|\kappa_{s}\right\|_{L^{2}\left(\Gamma_{t}\right)}^{2}+\left\{2 \mu^{*}-\widetilde{C}_{1}\left\|\kappa_{s}\right\|_{L^{2}\left(\Gamma_{t}\right)}^{2}-\widetilde{C}_{2}\left(\delta^{*}+\left|\kappa^{*}\right|\right)\left\|\kappa_{s}\right\|_{L^{2}\left(\Gamma_{t}\right)}\right\}\left\|V_{s}\right\|_{L^{2}\left(\Gamma_{t}\right)}^{2} \leq 0 .
$$

Then, we choose $\delta_{1}$ such that

$$
0<\delta_{1}<\min \left\{\frac{\mu^{*}}{2 \widetilde{C}_{1}},\left(\frac{\mu^{*}}{2 \widetilde{C}_{2}\left(\delta^{*}+\left|\kappa^{*}\right|\right)}\right)^{2}\right\} .
$$

Assuming $\left\|\kappa_{s}(t)\right\|_{L^{2}\left(\Gamma_{t}\right)}^{2}<\delta_{1}$ for $t \in(0, T]$, it follows that

$$
\frac{d}{d t}\left\|\kappa_{s}(t)\right\|_{L^{2}\left(\Gamma_{t}\right)}^{2}+\mu^{*}\left\|V_{s}(t)\right\|_{L^{2}\left(\Gamma_{t}\right)}^{2} \leq 0
$$

Integrating (4.8) with respect to $t$ in the interval $\left[t_{0}, t\right]$, we derive the desired result.

Now we arrive at the main result in this section.

Theorem 4.7 (Global existence) Let $\Gamma^{*}$ be a stationary curve such that the bilinear form $I^{*}$ is positive. Also, let $\rho_{0} \in C^{2+\alpha}(\mathcal{I})$ be a function describing a curve $\Gamma_{0}$, which is close to $\Gamma^{*}$ as in Section 2 and satisfies $\Gamma_{0} \perp \partial \Omega$. Assume that a curve $\Gamma_{0}$ includes the same area as $\Gamma^{*}$. Then, there exist constants $\gamma_{0}>0$ and $\delta_{0}>0$ such that if $\left\|\rho_{0}\right\|_{C^{1}(\mathcal{I})}<\gamma_{0}$ and $L\left[\Gamma_{0}\right]-L^{*}<\delta_{0}$, the problem (2.5) admits a unique global-in-time solution $\rho$ with

$$
\|\rho(t)\|_{C^{1}(\mathcal{I})}<\gamma_{0} \quad \text { and } \quad L\left[\Gamma_{t}\right]-L^{*}<\delta_{0} \quad \text { for } \quad t \geq 0
$$

where $\Gamma_{t}$ is the curve parameterized by $\Psi(\sigma, \rho(\sigma, t))$ in Section 2. 
Proof. Choose $\gamma_{0}$ and $\delta_{0}$ satisfying

$$
0<\gamma_{0}<\frac{\gamma^{*}}{2}, \quad 0<\delta_{0}<\frac{\delta_{1}^{*}}{2}
$$

where $\gamma^{*}$ is as in Lemma 4.4 and $\delta_{1}^{*}$ is as in (4.6). Assume that the initial curve $\Gamma_{0}$ satisfies $\left\|\rho_{0}\right\|_{C^{1}(\mathcal{I})}<\gamma_{0}$ and $L\left[\Gamma_{0}\right]-L^{*}<\delta_{0}$. Then Lemma 4.4 and an embedding result imply

$$
\left\|\rho_{0}\right\|_{C^{0}(\mathcal{I})} \leq C\left(L\left[\Gamma_{0}\right]-L^{*}\right)<C \delta_{0}
$$

Further, Lemma 4.5(i) implies that for $t>0$

$$
L\left[\Gamma_{t}\right]-L^{*} \leq L\left[\Gamma_{0}\right]-L^{*}<\delta_{0} .
$$

We now prove that $\left\|\kappa_{s}(t)\right\|_{L^{2}\left(\Gamma_{t}\right)}^{2}<\delta_{1}$ for each time $t$ in the existence interval of the solution, where $\delta_{1}$ is as in Proposition 4.6. Let $0<\beta<\alpha<1 / 2$. By Theorem 2.1, we can construct a unique local-in-time solution for $\rho_{0} \in C^{2+\beta}(\mathcal{I})$ and obtain the estimate

$$
\|\rho\|_{\mathcal{Y}\left(\overline{\mathcal{Q}_{0, T_{0}}}\right)} \leq K_{0}
$$

where $K_{0}$ is a constant, which depends on $\left\|\rho_{0}\right\|_{C^{2+\beta}(\mathcal{I})}$ increasingly, and $T_{0}$ is the local existence time, which depends on $1 /\left\|\rho_{0}\right\|_{C^{2+\beta}(\mathcal{I})}$ increasingly (for details, see Appendix). According to the interpolation inequality for Hölder spaces and (4.10), we have

$$
\left\|\rho_{0}\right\|_{C^{2+\beta}(\mathcal{I})} \leq C\left(\left\|\rho_{0}\right\|_{C^{0}(\mathcal{I})}\right)^{\frac{\alpha-\beta}{2+\alpha}}\left(\left\|\rho_{0}\right\|_{C^{2+\alpha}(\mathcal{I})}\right)^{\frac{2+\beta}{2+\alpha}} \leq \widetilde{C} \delta_{0}^{\frac{\alpha-\beta}{2+\alpha}}
$$

Set $t_{0}:=\delta_{0}^{\frac{\alpha-\beta}{2+\alpha}}>0$. Then it follows from (4.12), (4.13), and the definition of $\mathcal{Y}\left(\overline{\mathcal{Q}_{0, T_{0}}}\right)$ that there exist $C>0$ and $\nu>0$ such that

$$
\left\|\kappa_{s}\left(t_{0}\right)\right\|_{L^{2}\left(\Gamma_{t}\right)}^{2} \leq C \delta_{0}^{\nu}
$$

Since $\|\rho(t)\|_{C^{1}(\mathcal{I})}$ is continuous with respect to $t$ until $t=0$, we see that $\|\rho(t)\|_{C^{1}(\mathcal{I})}<\gamma^{*}$ for $t \in[0, T]$ with a $T \in\left(0, T_{0}\right]$. Further, by (4.9) and (4.11), we have $L\left[\Gamma_{t}\right]-L^{*}<\delta_{1}^{*}$ for $t>0$. Choose $\delta_{0}$ such that $t_{0}<T$ and $C \delta_{0}^{\nu}<\delta_{1}$. Then, by applying a similar argument to [7, Proof of Theorem 6.1] together with Proposition 4.6, we obtain that $\left\|\kappa_{s}(t)\right\|_{L^{2}\left(\Gamma_{t}\right)}^{2}<\delta_{1}$ for $t \in\left[t_{0}, T\right]$.

Next, we prove that $\|\rho(t)\|_{C^{1}(\mathcal{I})}<\gamma_{0}$ for $t \in\left[t_{0}, T\right]$. By Lemma 4.4 and (4.11), it holds that for $t \in[0, T]$

$$
\bar{c}\|\rho(t)\|_{H^{1}(\mathcal{I})} \leq L\left[\Gamma_{t}\right]-L^{*}<\delta_{0} .
$$

Then, by the embedding inequality and (4.14), we see that $\|\rho(t)\|_{C^{0}(\mathcal{I})} \leq C \delta_{0}$ for $t \in[0, T]$. On the other hand, it follows from Lemma 4.2(ii) and Lemma 4.5(ii) that there exists $C>0$ such that for $t \in\left[t_{0}, T\right]$

$$
\|\kappa(t)\|_{C^{0}\left(\Gamma_{t}\right)} \leq\left\|\kappa(t)-\kappa_{a v}(t)\right\|_{C^{0}\left(\Gamma_{t}\right)}+\left|\kappa_{a v}(t)-\kappa^{*}\right|+\left|\kappa^{*}\right| \leq C\left(\delta_{1}+\delta_{0}\right)+\left|\kappa^{*}\right| .
$$

Thus, by virtue of (4.14), (4.15), and $\left\|\kappa_{s}(t)\right\|_{L^{2}\left(\Gamma_{t}\right)}^{2}<\delta_{1}$ for $t \in\left[t_{0}, T\right]$, we derive the boundedness of $\|\rho(t)\|_{H^{3}(\mathcal{I})}$ for $t \in\left[t_{0}, T\right]$, which implies the boundedness of $\|\rho(t)\|_{C^{2+\alpha}(\mathcal{I})}$ for $\alpha \in(0,1 / 2)$. Then, by the interpolation inequality for Hölder spaces, we have

$$
\|\rho(t)\|_{C^{1}(\mathcal{I})} \leq C\left(\|\rho(t)\|_{C^{0}(\mathcal{I})}\right)^{\frac{1+\alpha}{2+\alpha}}\left(\|\rho(t)\|_{C^{2+\alpha}(\mathcal{I})}\right)^{\frac{1}{2+\alpha}} \leq \widetilde{C} \delta_{0}^{\frac{1+\alpha}{2+\alpha}}
$$


for $t \in\left[t_{0}, T\right]$. Choosing $\delta_{0}$ such that $\widetilde{C} \delta_{0}^{\frac{1+\alpha}{2+\alpha}}<\gamma_{0}$, we obtain $\|\rho(t)\|_{C^{1}(\mathcal{I})}<\gamma_{0}$ for $t \in\left[t_{0}, T\right]$.

Finally, let us derive the existence of a unique global-in-time solution. Repeating the above argument until the local existence time $T_{0}$, we see that $\Gamma_{t}$ satisfies

$$
\|\rho(t)\|_{C^{1}(\mathcal{I})}<\gamma_{0}, \quad L\left[\Gamma_{t}\right]-L^{*}<\delta_{0}, \quad\left\|\kappa_{s}(t)\right\|_{L^{2}\left(\Gamma_{t}\right)}^{2}<\delta_{1}
$$

for $t \in\left[t_{0}, T_{0}\right]$. This implies that $\Gamma_{T_{0}}$ satisfies the same conditions as those fulfilled by $\Gamma_{0}$ and the boundedness of $\left\|\rho\left(T_{0}\right)\right\|_{C^{2+\alpha}(\mathcal{I})}$ for $\alpha \in(0,1 / 2)$ is guaranteed. Thus, due to Theorem 2.1, the solution of (2.5) can be extended over $t=T_{0}$ by a fixed amount of time. Further, by applying the same argument as we did in the first half of this proof, we have the estimates (4.16) for each time $t$ in the extended existence interval of the solution. This procedure can be iterated as many times as we want, so that a unique global-in-time solution of $(2.5)$ with $\rho(\cdot, 0)=\rho_{0}$ can be obtained.

\section{$5 \quad$ Stability of stationary curves}

The following theorem shows nonlinear stability of the stationary curve $\Gamma^{*}$ when the bilinear form $I^{*}$ is positive.

Theorem 5.1 (Nonlinear stability) Let the assumption of Theorem 4.7 hold. Then

$$
\|\rho(t)\|_{H^{3}(\mathcal{I})} \rightarrow 0 \quad \text { as } \quad t \rightarrow \infty
$$

Proof. We apply a method similar to the one used in [7, Proof of Theorem 6.4]. By Lemma 4.1(i), we see

$$
\int_{0}^{\infty}\left\|\kappa_{s}(\tau)\right\|_{L^{2}\left(\Gamma_{\tau}\right)}^{2} d \tau \leq L\left[\Gamma_{0}\right]
$$

This implies that for any $\varepsilon \in\left(0, \delta_{1}\right)$ there exists a sufficiently large $t_{\varepsilon}>0$ such that

$$
\left\|\kappa_{s}\left(t_{\varepsilon}\right)\right\|_{L^{2}\left(\Gamma_{t}\right)}^{2}<\varepsilon
$$

According to the proof of Theorem 4.7, it holds $\left\|\kappa_{s}(t)\right\|_{L^{2}\left(\Gamma_{t}\right)}^{2}<\delta_{1}$ as long as the solution exists. Thus, applying Proposition 4.6 for $t \in\left[t_{\varepsilon}, \infty\right)$, we have

$$
\left\|\kappa_{s}(t)\right\|_{L^{2}\left(\Gamma_{t}\right)}^{2}+\mu^{*} \int_{t_{\varepsilon}}^{t}\left\|V_{s}(\tau)\right\|_{L^{2}\left(\Gamma_{t}\right)}^{2} d \tau \leq\left\|\kappa_{s}\left(t_{\varepsilon}\right)\right\|_{L^{2}\left(\Gamma_{t}\right)}^{2}<\varepsilon .
$$

This means that

$$
\left\|\kappa_{s}(t)\right\|_{L^{2}\left(\Gamma_{t}\right)}^{2} \rightarrow 0 \quad \text { as } \quad t \rightarrow \infty
$$

By (5.1) and Lemma 4.2(ii), we also see

$$
\left\|\kappa(\cdot, t)-\kappa_{a v}(t)\right\|_{C^{0}\left(\Gamma_{t}\right)} \rightarrow 0 \quad \text { as } \quad t \rightarrow \infty .
$$

On the other hand, by virtue of Lemma 4.4 and Lemma 4.5(i), we obtain the boundedness of $\|\rho(t)\|_{H^{1}(\mathcal{I})}$. Using Lemma 4.5 and (5.2), we also have the boundedness of 
$\|\kappa(t)\|_{L^{2}\left(\Gamma_{t}\right)}$. Then, the boundedness of $\|\rho(t)\|_{H^{1}(\mathcal{I})}$ and $\|\kappa(t)\|_{L^{2}\left(\Gamma_{t}\right)}$ imply the boundedness of $\|\rho(t)\|_{H^{2}(\mathcal{I})}$. Since it follows from the boundedness of $\|\rho(t)\|_{H^{2}(\mathcal{I})}$ and (5.1) that $\|\rho(t)\|_{H^{3}(\mathcal{I})}$ is bounded, there exists a sequence $\left\{t_{n}\right\}_{n \in \mathbb{N}}$ and $\widetilde{\rho}$ such that

$$
\rho\left(t_{n}\right) \rightarrow \widetilde{\rho} \quad \text { in } \quad C^{2+\alpha}(\mathcal{I}) \quad \text { as } \quad n \rightarrow \infty
$$

By virtue of (5.2), $\widetilde{\rho}$ satisfies $\widetilde{\kappa}-\widetilde{\kappa}_{a v}=0$. The solution of the problem

$$
\kappa=\kappa_{a v}, \quad \varangle(\Gamma, \partial \Omega)=\pi / 2, \quad \text { Area }[\Gamma]=\text { Area }\left[\Gamma^{*}\right]
$$

is unique in the $C^{0}$-neighborhood of $\Gamma^{*}$ and given by $\rho \equiv 0$ (see Theorem 5.2 below). Since $\widetilde{\rho}$ is a solution of this problem, we obtain $\widetilde{\rho} \equiv 0$. In particular, we get

$$
L\left[\Gamma_{t_{n}}\right] \rightarrow L\left[\Gamma^{*}\right]=L^{*} \quad \text { as } \quad n \rightarrow \infty .
$$

We remark that $\Gamma_{t_{n}}$ and $\Gamma^{*}$ are the curves described by $\rho=\rho\left(t_{n}\right)$ and $\rho \equiv 0$ for the parameterization in Section 2, respectively. Then, by the fact that $L\left[\Gamma_{t}\right]$ decreases in time, we obtain that

$$
L\left[\Gamma_{t}\right] \rightarrow L^{*} \quad \text { as } \quad t \rightarrow \infty .
$$

Applying Lemma 4.4, we have

$$
\|\rho(t)\|_{H^{1}(\mathcal{I})}^{2} \rightarrow 0 \quad \text { as } \quad t \rightarrow \infty .
$$

Hence, using this fact together with both (5.1) and (5.2), we obtain the desired result.

It remains to prove the following result. We refer to Grosse-Brauckmann [16] for a similar proof in the case of a different boundary condition.

Theorem 5.2 Let $\Gamma^{*}$ be a stationary curve such that the bilinear form $I^{*}$ is positive and let $\Gamma$ be a curve described by $\rho$ for the parameterization in Section 2. Then there exists a $C^{2}$-neighborhood of $\Gamma^{*}$ such that $\rho \equiv 0$ is the unique solution of the problem

$$
\kappa=\kappa_{a v}, \quad \varangle(\Gamma, \partial \Omega)=\pi / 2, \quad \text { Area }[\Gamma]=\text { Area }\left[\Gamma^{*}\right] .
$$

Proof. We use the following implicit function theorem (see Zeidler [23, Theorem 4.B]).

\section{Suppose that}

(i) the mapping $F: U\left(x_{0}, y_{0}\right) \subset X \times Y \rightarrow Z$ is defined on an open neighbourhood $U\left(x_{0}, y_{0}\right)$ of $\left(x_{0}, y_{0}\right)$, and $F\left(x_{0}, y_{0}\right)=0$, where $X, Y$ and $Z$ are Banach spaces over $\mathbb{R}$.

(ii) $F_{y}$ exists as partial Fréchet derivative on $U\left(x_{0}, y_{0}\right)$ and

$$
F_{y}\left(x_{0}, y_{0}\right): Y \rightarrow Z
$$

is bijective.

(iii) $F$ and $F_{y}$ are continuous at $\left(x_{0}, y_{0}\right)$. 
Then the following holds true: There exist positive numbers $r_{0}$ and $r$ such that, for every $x \in X$ satisfying $\left\|x-x_{0}\right\|<r_{0}$, there is exactly one $y(x) \in Y$ for which $\left\|y(x)-y_{0}\right\| \leq r$ and $F(x, y(x))=0$.

We use this theorem for

$$
\begin{aligned}
X & :=\left\{\rho \in C^{2}(\mathcal{I}) \mid \rho=\text { const. }\right\} \\
Y & :=\left\{\rho \in C^{2}(\mathcal{I}) \mid \int_{l_{-}}^{l_{+}} \rho d \sigma=0\right\}, \\
Z & :=\left\{\rho \in C^{0}(\mathcal{I}) \mid \int_{l_{-}}^{l_{+}} \rho d \sigma=0\right\} \times \mathbb{R}^{2}
\end{aligned}
$$

and

$$
F(m, u):=\left(\kappa-\kappa_{a v}, \varangle\left(\partial \Omega, \Gamma_{t}\right)_{+}-\frac{\pi}{2}, \varangle\left(\partial \Omega, \Gamma_{t}\right)_{-}-\frac{\pi}{2}\right)
$$

where $\kappa$ is computed for the curve that we get by taking $\rho=u+m$ in Section 2. The expression $\varangle\left(\partial \Omega, \Gamma_{t}\right)_{ \pm}$denotes the angles with the outer boundary at the two boundary points. The derivative $F_{u}(0,0)$ is (by a similar computation as in [12]) given by

$F_{u}(0,0)(v)=\left(\left(\partial_{\sigma}^{2}+\kappa_{1}^{2}\right) v-\frac{1}{l_{+}-l_{-}} \int_{l_{-}}^{l_{+}}\left(\partial_{\sigma}^{2}+\kappa_{\sigma}^{2}\right) v d \sigma,\left(\partial_{\sigma}+h_{+}\right) v\left(l_{+}\right),\left(\partial_{\sigma}-h_{-}\right) v\left(l_{-}\right)\right)$.

The fact that $I^{*}$ is positive implies that $F_{u}(0,0)$ is invertible (using regularity theory for ordinary differential equations). Straightforward computations show that $F$ and $F_{u}$ are continuous at $(0,0)$.

Hence, for $m \in X$ small we find exactly one $u(m)$ such that

$$
F(m, u(m))=0 .
$$

Let us define

$$
\rho_{m}=u(m)+m
$$

and let $\Gamma_{m}$ be a curve described by $\rho_{m}$ for the parameterization in Section 2 . Then we have

$$
\begin{aligned}
\operatorname{Area}\left[\Gamma_{m}\right] & =\text { Area }\left[\Gamma^{*}\right]+\int_{l_{-}}^{l_{+}}(u(m)+m) d \sigma+\mathcal{O}\left(\|u(m)+m\|_{C^{2}(\mathcal{I})}^{2}\right) \\
& =\text { Area }\left[\Gamma^{*}\right]+\left(l_{+}-l_{-}\right) m+\mathcal{O}\left(\|u(m)+m\|_{C^{2}(\mathcal{I})}^{2}\right) .
\end{aligned}
$$

This implies that for $m \neq 0$

$$
\left|\operatorname{Area}\left[\Gamma_{m}\right]-\operatorname{Area}\left[\Gamma^{*}\right]\right| \neq 0,
$$

if $\|(m, u(m))\|_{C^{2}(\mathcal{I})}$ is small enough. We now represent a solution $\rho$ of (5.3) with $\|\rho\|_{C^{2}(\mathcal{I})}$ small as $\rho=u+m$ where $u=\rho-\rho_{a v}$ and $m=\rho_{a v}$ with

$$
\rho_{a v}=\frac{1}{l_{+}-l_{-}} \int_{l_{-}}^{l_{+}} \rho d \sigma .
$$

Then we see $F(m, u)=0$. Due to the area-preserving property and (5.4), we obtain $m=0$ and $u \equiv 0$ which implies $\rho \equiv 0$. This proves the theorem. 


\section{A Proof of Theorem 2.1}

The problem (2.5) is an initial boundary value problem for a quasilinear parabolic partial differential equation which has the form

$$
\begin{cases}\rho_{t}=-\frac{1}{(J(\rho))^{4}} \partial_{\sigma}^{4} \rho+a\left(\rho, \partial_{\sigma} \rho, \partial_{\sigma}^{2} \rho\right) \partial_{\sigma}^{3} \rho+f\left(\rho, \partial_{\sigma} \rho, \partial_{\sigma}^{2} \rho\right) & \text { in } \mathcal{Q}_{0, T} \\ b_{1}(\rho) \partial_{\sigma} \rho+g_{1}(\rho)=0 & \text { at } \sigma=l_{ \pm} \\ b_{2}\left(\rho, \partial_{\sigma} \rho\right) \partial_{\sigma}^{3} \rho+g_{2}\left(\rho, \partial_{\sigma} \rho, \partial_{\sigma}^{2} \rho\right)=0 & \text { at } \sigma=l_{ \pm} \\ \left.\rho\right|_{t=0}=\rho_{0} & \text { in } \mathcal{I},\end{cases}
$$

where $a, f, b_{i}$, and $g_{i}(i=1,2)$ are smooth functions with respect to $\rho, \partial_{\sigma} \rho$, and $\partial_{\sigma}^{2} \rho$; and $g_{i}(i=1,2)$ satisfy $\left\|g_{1}(t)\right\|_{C^{0}(\mathcal{I})}=\mathcal{O}\left(\|\rho(t)\|_{C^{0}(\mathcal{I})}\right)$ and $\left\|g_{2}(t)\right\|_{C^{0}(\mathcal{I})}=\mathcal{O}\left(\|\rho(t)\|_{C^{2+\alpha}(\mathcal{I})}\right)$ when $\|\rho\|_{C^{2+\alpha}(\mathcal{I})} \rightarrow 0$. In order to prove Theorem 2.1, we apply a fixed point argument. Let

$$
\mathcal{D}:=\left\{\rho \in \mathcal{Y}\left(\overline{\mathcal{Q}_{0, T}}\right) \mid \rho(\cdot, 0)=\rho_{0},\|\rho\|_{\mathcal{Y}\left(\overline{\mathcal{Q}_{0, T}}\right)} \leq K\right\}
$$

for positive constants $K$ and $T$, and define a mapping $\mathcal{P}$ as

$$
\mathcal{P}: \mathcal{D} \ni \bar{\rho} \mapsto \rho \in \mathcal{Y}\left(\overline{\mathcal{Q}_{0, T}}\right)
$$

where $\rho$ is the unique solution of the linearized problem

$$
\begin{cases}\rho_{t}=\mathcal{A} \rho+F(\sigma, t) & \text { for } \quad(\sigma, t) \in \mathcal{Q}_{0, T}, \\ \mathcal{B}_{1} \rho=G_{1}(\sigma, t) & \text { at } \quad \sigma=l_{ \pm}, t \in(0, T], \\ \mathcal{B}_{2} \rho=G_{2}(\sigma, t) & \text { at } \quad \sigma=l_{ \pm}, t \in(0, T], \\ \rho(\sigma, 0)=\rho_{0} & \text { for } \quad \sigma \in \mathcal{I} .\end{cases}
$$

Here the linearized operators $\mathcal{A}, \mathcal{B}_{1}$, and $\mathcal{B}_{2}$ around the initial data $\rho_{0} \in C^{2+\alpha}(\mathcal{I})$ are given by

$$
\begin{aligned}
& \mathcal{A}=-\frac{1}{\left(J\left(\rho_{0}\right)\right)^{4}} \partial_{\sigma}^{4}+a\left(\rho_{0}, \partial_{\sigma} \rho_{0}, \partial_{\sigma}^{2} \rho_{0}\right) \partial_{\sigma}^{3}, \\
& \mathcal{B}_{1}=b_{1}\left(\rho_{0}\right) \partial_{\sigma}, \quad \mathcal{B}_{2}=b_{2}\left(\rho_{0}, \partial_{\sigma} \rho_{0}\right) \partial_{\sigma}^{3}
\end{aligned}
$$

and for given $\bar{\rho} \in \mathcal{D}$

$$
\begin{aligned}
F(\sigma, t)= & -\left\{\frac{1}{(J(\bar{\rho}))^{4}}-\frac{1}{\left(J\left(\rho_{0}\right)\right)^{4}}\right\} \partial_{\sigma}^{4} \bar{\rho} \\
& +\left\{a\left(\bar{\rho}, \partial_{\sigma} \bar{\rho}, \partial_{\sigma}^{2} \bar{\rho}\right)-a\left(\rho_{0}, \partial_{\sigma} \rho_{0}, \partial_{\sigma}^{2} \rho_{0}\right)\right\} \partial_{\sigma}^{3} \bar{\rho} \\
& +f\left(\bar{\rho}, \partial_{\sigma} \bar{\rho}, \partial_{\sigma}^{2} \bar{\rho}\right), \\
G_{1}(\sigma, t)= & -\left\{b_{1}(\bar{\rho})-b_{1}\left(\rho_{0}\right)\right\} \partial_{\sigma} \bar{\rho}-g_{1}(\bar{\rho}), \\
G_{2}(\sigma, t)= & -\left\{b_{2}\left(\bar{\rho}, \partial_{\sigma} \bar{\rho}\right)-b_{2}\left(\rho_{0}, \partial_{\sigma} \rho_{0}\right)\right\} \partial_{\sigma}^{3} \bar{\rho}-g_{2}\left(\bar{\rho}, \partial_{\sigma} \bar{\rho}, \partial_{\sigma}^{2} \bar{\rho}\right) .
\end{aligned}
$$

The existence of a unique solution for the linearized problem (A.2) in $\mathcal{Y}\left(\overline{\mathcal{Q}_{0, T}}\right)$ is proved by applying the optimal regularity theory for analytic semigroups to the linearized problem (A.2) (see [17]). If the mapping $\mathcal{P}$ is a contraction on $\mathcal{D}$ for suitable constants $K$ and $T$ 
depending on $\left\|\rho_{0}\right\|_{C^{2+\alpha}(\mathcal{I})}, \mathcal{P}$ has a unique fixed point in $\mathcal{D}$ which is a unique solution of the nonlinear problem (A.1). Thus we show that the mapping $\mathcal{P}$ is a contraction on $\mathcal{D}$. In order to prove this fact, the following lemma is crucial.

Lemma A.1 (i) Assume that $\bar{\rho} \in \mathcal{D}$ and that $\rho$ is a solution of the linearized problem (A.2). Then there exist positive constants $M_{0}$ and $N$ such that

$$
\|\rho\|_{\mathcal{Y}\left(\overline{\mathcal{Q}_{0, T}}\right)} \leq M_{0}+N T^{\frac{\alpha}{4}}
$$

In particular, $M_{0}$ depends on $\left\|\rho_{0}\right\|_{C^{2+\alpha}(\mathcal{I})}$ increasingly, and $N$ depends on $K$ increasingly. (ii) Assume that $\bar{\rho}_{1}, \bar{\rho}_{2} \in \mathcal{D}$ and that $\rho_{1}, \rho_{2}$ are solutions of the linearized problem (A.2). Then there exists a positive constant $N$ such that

$$
\left\|\rho_{1}-\rho_{2}\right\|_{\mathcal{Y}\left(\overline{\mathcal{Q}_{0, T}}\right)} \leq N T^{\frac{\alpha}{4}}\left\|\bar{\rho}_{1}-\bar{\rho}_{2}\right\|_{\mathcal{Y}\left(\overline{\mathcal{Q}_{0, T}}\right)} .
$$

In particular, $N$ depends on $K$ increasingly.

A method to prove this lemma is to use the optimal regularity theory of analytic semigroups as in [17]. We prove this lemma in the next section.

Lemma A.1 implies that if we take

$$
K=2 M_{0}, \quad T_{0}=\min \left\{\left(\frac{K}{2 N}\right)^{4 / \alpha},\left(\frac{1}{2 N}\right)^{4 / \alpha}\right\},
$$

it follows that for $T \leq T_{0}$

$$
\|\rho\|_{\mathcal{Y}\left(\overline{\mathcal{Q}_{0, T}}\right)} \leq K, \quad\left\|\rho_{1}-\rho_{2}\right\|_{\mathcal{Y}\left(\overline{\mathcal{Q}_{0, T}}\right)} \leq \frac{1}{2}\left\|\bar{\rho}_{1}-\bar{\rho}_{2}\right\|_{\mathcal{Y}\left(\overline{\mathcal{Q}_{0, T}}\right)} .
$$

This means that $\mathcal{P}$ maps $\mathcal{D}$ into itself and is a contraction on $\mathcal{D}$ for $T \leq T_{0}$. Thus the proof of Theorem 2.1 is completed.

\section{B Proof of Lemma A.1}

We only prove Lemma A.1(i). Applying a similar argument, we can also derive Lemma A.1(ii). It is convenient to introduce the following estimate without proof.

Lemma B.1 (see [17, Section 2]) For $k \in \mathbb{N}, \beta_{1}, \beta_{2} \in(0,1)$, and a sectorial operator $A$, there exists a constant $C=C\left(k, \beta_{1}, \beta_{2}, A\right)$ such that

$$
\left\|t^{k-\beta_{1}+\beta_{2}} A^{k} e^{t A}\right\|_{L\left(D_{A}\left(\beta_{1}, \infty\right), D_{A}\left(\beta_{2}, \infty\right)\right)} \leq C \text { for } 0<t \leq 1 .
$$

The statement holds also for $k=0$, provided $\beta_{1} \leq \beta_{2}$.

Define $X:=C(\mathcal{I})$ and

$$
D(A):=\left\{u \in C^{4}(\mathcal{I}) \mid \mathcal{B}_{1} u\left(l_{ \pm}\right)=\mathcal{B}_{2} u\left(l_{ \pm}\right)=0\right\} .
$$

Then $A: X \supset D(A) \ni u \mapsto \mathcal{A} u \in X$ is the realization of $\mathcal{A}$ in $X$. It is known that $A$ is a sectorial operator in $X$ (see $[20]$ ). 
Let $\rho$ be a unique solution of the linearized problem (A.2). In order to reduce the inhomogeneous problem to a homogeneous problem at the boundaries, we introduce an auxiliary function $\zeta$ defined as

$$
\begin{aligned}
\zeta(\sigma, t):= & \left\{\frac{\left(\sigma-l_{-}\right) G_{1}\left(l_{-}, t\right)}{\left.b_{1}\left(\rho_{0}\right)\right|_{\sigma=l_{-}}}+\frac{\left(\sigma-l_{-}\right)^{3} G_{2}\left(l_{-}, t\right)}{\left.3 ! b_{2}\left(\rho_{0}, \partial_{\sigma} \rho_{0}\right)\right|_{\sigma=l_{-}}}\right\} \eta(\sigma) \\
& +\left\{\frac{\left(\sigma-l_{+}\right) G_{1}\left(l_{+}, t\right)}{\left.b_{1}\left(\rho_{0}\right)\right|_{\sigma=l_{+}}}+\frac{\left(\sigma-l_{+}\right)^{3} G_{2}\left(l_{+}, t\right)}{\left.3 ! b_{2}\left(\rho_{0}, \partial_{\sigma} \rho_{0}\right)\right|_{\sigma=l_{+}}}\right\} \hat{\eta}(\sigma)
\end{aligned}
$$

where $\eta, \hat{\eta} \in C^{\infty}(\mathcal{I})$ are cut-off functions satisfying

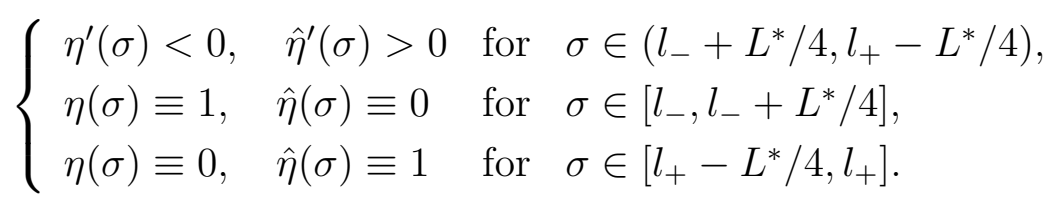

Then it follows that $\rho-\zeta$ fulfills homogeneous boundary conditions. Since $A$ is sectorial, we represent $\rho-\zeta$ with the help of a variant of the variation of constants formula and the analytic semigroup $e^{t A}$. By a simple computation, we obtain for $0 \leq t \leq T$,

$$
\rho(\cdot, t)=\rho_{1}(\cdot, t)+\rho_{2}(\cdot, t)+\rho_{3}(\cdot, t)
$$

where

$$
\begin{aligned}
& \rho_{1}(\cdot, t)=e^{t A}\left\{\rho_{0}-\zeta(\cdot, 0)\right\}, \\
& \rho_{2}(\cdot, t)=\int_{0}^{t} e^{(t-r) A}\{F(\cdot, r)+\mathcal{A} \zeta(\cdot, r)\} d r \\
& \rho_{3}(\cdot, t)=-A \int_{0}^{t} e^{(t-r) A}\{\zeta(\cdot, r)-\zeta(\cdot, 0)\} d r+\zeta(\cdot, 0) .
\end{aligned}
$$

Applying the theory of analytic semigroups as in [17], we have (see below)

$$
\left\{\begin{aligned}
&\left\|\rho_{1}\right\|_{\mathcal{Y}\left(\overline{\mathcal{Q}_{0, T}}\right)} \leq C_{0}\left\|\rho_{0}-\zeta(\cdot, 0)\right\|_{D_{A}\left(\frac{2+\alpha}{4}, \infty\right)} \\
&\left\|\rho_{2}\right\|_{\mathcal{Y}\left(\overline{\mathcal{Q}_{0, T}}\right)} \leq C_{0} \sup _{0<\delta<T} \delta^{\frac{1}{2}} \sup _{t \in[\delta, T]}\|F(\cdot, t)+\mathcal{A} \zeta(\cdot, t)\|_{D_{A}\left(\frac{\alpha}{4}, \infty\right)} \\
&\left\|\rho_{3}\right\|_{\mathcal{Y}\left(\overline{\mathcal{Q}_{0, T}}\right)} \leq C_{0}+C_{0, K} T^{\frac{1}{4}}
\end{aligned}\right.
$$

In particular, it is verified that a constant $C_{0}$ increases with $\left\|\rho_{0}\right\|_{C^{2+\alpha}(\mathcal{I})}$, and that a constant $C_{0, K}$ increases with $\left\|\rho_{0}\right\|_{C^{2+\alpha}(\mathcal{I})}$ and $K$. Once (B.2) is proven, it follows from characterization of interpolation spaces $D_{A}(\beta, \infty)$ (see e.g. $\left.[1,17,18]\right)$ and the definition of $F$ that

$$
\begin{aligned}
\|\rho\|_{\mathcal{Y}\left(\overline{\mathcal{Q}_{0, T}}\right)} \leq & \left\|\rho_{1}\right\|_{\mathcal{Y}\left(\overline{\mathcal{Q}_{0, T}}\right)}+\left\|\rho_{2}\right\|_{\mathcal{Y}\left(\overline{\mathcal{Q}_{0, T}}\right)}+\left\|\rho_{3}\right\|_{\mathcal{Y}\left(\overline{\mathcal{Q}_{0, T}}\right)} \\
\leq & \widetilde{C}_{0}\left\|\rho_{0}-\zeta(\cdot, 0)\right\|_{C^{2+\alpha}(\mathcal{I})} \\
& +\widetilde{C}_{0} \sup _{0<\delta<T} \delta^{\frac{1}{2}} \sup _{t \in[\delta, T]}\|F(\cdot, t)+\mathcal{A} \zeta(\cdot, t)\|_{C^{\alpha}(\mathcal{I})} \\
& +\widetilde{C}_{0}+\widetilde{C}_{0, K} T^{\frac{1}{4}} \\
\leq & M_{0}+N_{0, K} T^{\frac{\alpha}{4}}+N_{0, K} T^{\frac{1}{4}}
\end{aligned}
$$


where $\widetilde{C}_{0}$ and $M_{0}$ depend on $\left\|\rho_{0}\right\|_{C^{2+\alpha}(\mathcal{I})}$ increasingly, and $\widetilde{C}_{0, K}$ and $N_{0, K}$ depend on $\left\|\rho_{0}\right\|_{C^{2+\alpha}(\mathcal{I})}$ and $K$ increasingly. This completes the proof of Lemma A.1(i). Thus we give the proof of (B.2) in detail.

First let us explain about the estimates for $\rho_{1}$ and $\rho_{2}$. Using (B.1) with $k=0$ and $\beta_{1}=\beta_{2}=(2+\alpha) / 4$ to $\rho_{1}$, and with $k=1, \beta_{1}=(2+\alpha) / 4$, and $\beta_{2}=\alpha / 4$ to $\partial \rho_{1} / \partial t=A \rho_{1}$, we are led to the estimate of $\rho_{1}$ easily. Since $F+\mathcal{A} \zeta \in L^{\infty}\left((0, T] ; D_{A}\left(\frac{\alpha}{4}, \infty\right)\right)$, applying the same argument as $\left[17\right.$, Section 4.3.2] to $\rho_{2}$ in $[\varepsilon, T](\varepsilon \in(0, T))$, we have an estimate for $\rho_{2}$. Let us consider the estimate for $\rho_{3}$. Since $\zeta$ is less regular, we cannot derive the desired estimate for $\rho_{3}$ if we only use (B.1) to $\rho_{3}$ directly. Set

$$
z(t)=\int_{0}^{t} e^{(t-r) A}\{\zeta(\cdot, r)-\zeta(\cdot, 0)\} d r
$$

Then $z$ satisfies

$$
\begin{aligned}
& \rho_{3}(\cdot, t)=-A z(t)+\zeta(\cdot, 0)=-\frac{d}{d t} z(t)+\zeta(\cdot, t), \\
& \frac{d}{d t} \rho_{3}(\cdot, t)=-A \frac{d}{d t} z(t)=A\left\{\rho_{3}(\cdot, t)-\zeta(\cdot, t)\right\} .
\end{aligned}
$$

This means that if we obtain the estimates for $d z / d t$, we have the desired estimates for $\rho_{3}$. In fact, the estimate for $\left\|\rho_{3}\right\|_{\mathcal{Y}\left(\overline{\mathcal{Q}_{0, T}}\right)}$ is given by

$$
\begin{aligned}
\left\|\rho_{3}\right\|_{\mathcal{Y}\left(\overline{\mathcal{Q}_{0, T}}\right)} \leq & \|\zeta(\cdot, 0)\|_{C^{2+\alpha}\left(\overline{\mathcal{Q}_{0, T}}\right)}+\|\zeta(\cdot, t)-\zeta(\cdot, 0)\|_{C^{2+\alpha}\left(\overline{\mathcal{Q}_{0, T}}\right)} \\
& +\sum_{i=1}^{3} \sup _{0<t<T} t^{\frac{1}{2}}\|\mathcal{A} \zeta(\cdot, t)\|_{C^{\alpha}\left(\overline{\mathcal{Q}_{0, T}}\right)} \\
& +\widetilde{C}\left(\|\dot{z}(t)\|_{D_{A}\left(\frac{2+\alpha}{4}, \infty\right)}+\sup _{0<\delta<T} \delta^{\frac{1}{2}} \sup _{t \in[\delta, T]}\|A \dot{z}(t)\|_{D_{A}\left(\frac{\alpha}{4}, \infty\right)}\right) .
\end{aligned}
$$

Here and hereafter we use $\dot{z}$ instead of $d z / d t$ to simplify the notation. For the function $z$, we have the following estimates.

Lemma B.2 Let $z$ be a function represented by (B.3). Then, there exists a constant $N$, which depends on $\left\|\rho_{0}\right\|_{C^{2+\alpha}(\mathcal{I})}$, $\alpha$, and $K$, such that

$$
\left\{\begin{array}{l}
\|\dot{z}(t)\|_{D_{A}\left(\frac{2+\alpha}{4}, \infty\right)} \leq N T^{\frac{1}{4}} \\
\sup _{0<\delta<T} \delta^{\frac{1}{2}} \sup _{t \in[\delta, T]}\|A \dot{z}(t)\|_{D_{A}\left(\frac{\alpha}{4}, \infty\right)} \leq N T^{\frac{1}{4}}
\end{array}\right.
$$

Proof. The proof of the first estimate of (B.4) is similar to arguments in [13, Appendix]. We only prove the second estimate of (B.4). For $t \geq \varepsilon$ with $\varepsilon \in(0, T)$, we have

$$
\begin{aligned}
\dot{z}(t)= & e^{(t-\varepsilon / 2) A} \dot{z}(\varepsilon / 2)+\int_{\varepsilon / 2}^{t} A e^{(t-r) A}\{\zeta(\cdot, r)-\zeta(\cdot, t)\} d r \\
& +e^{(t-\varepsilon / 2) A}\{\zeta(\cdot, t)-\zeta(\cdot, \varepsilon / 2)\} .
\end{aligned}
$$


This implies that

$$
\begin{aligned}
\|A \dot{z}(t)\|_{D_{A}\left(\frac{\alpha}{4}, \infty\right) \leq} \leq & \left\|A e^{(t-\varepsilon / 2) A} \dot{z}(\varepsilon / 2)\right\|_{D_{A}\left(\frac{\alpha}{4}, \infty\right)} \\
& +\left\|\int_{\varepsilon / 2}^{t} A^{2} e^{(t-r) A}\{\zeta(\cdot, r)-\zeta(\cdot, t)\} d r\right\|_{D_{A}\left(\frac{\alpha}{4}, \infty\right)} \\
& +\left\|A e^{(t-\varepsilon / 2) A}\{\zeta(\cdot, t)-\zeta(\cdot, \varepsilon / 2)\}\right\|_{D_{A}\left(\frac{\alpha}{4}, \infty\right)} \\
=: & I_{1}(t)+I_{2}(t)+I_{3}(t) .
\end{aligned}
$$

Let us first derive the estimate of $I_{1}(t)$. It follows that for $t \geq \varepsilon$

$$
I_{1}(t) \leq C_{0}(t-\varepsilon / 2)^{-\frac{\alpha}{4}}\|A \dot{z}(\varepsilon / 2)\| \leq C_{0}(\varepsilon / 2)^{-\frac{\alpha}{4}}\|A \dot{z}(\varepsilon / 2)\| .
$$

Thus it is necessary to obtain an estimate of $\|A \dot{z}(t)\|$. Since $\dot{z}(0)=0$, we see

$$
\|A \dot{z}(t)\| \leq \int_{0}^{t}\left\|A^{2} e^{(t-r) A}\{\zeta(\cdot, r)-\zeta(\cdot, t)\}\right\| d r+\left\|A e^{t A}\{\zeta(\cdot, t)-\zeta(\cdot, 0)\}\right\| .
$$

We now recall the definition of $\zeta$. Then we have to estimate each term. We show the estimate only for the term including the function

$$
\hat{\zeta}(\sigma, t):=\left(\sigma-l_{-}\right)^{3} G_{2}\left(l_{-}, t\right) \eta(\sigma)
$$

The ideas for the estimation of the other terms is similar. Set

$$
\begin{aligned}
J_{1}(t) & :=\int_{0}^{t}\left\|A^{2} e^{(t-r) A}\{\hat{\zeta}(\cdot, \sigma)-\hat{\zeta}(\cdot, t)\}\right\| d r \\
J_{2}(t) & :=\left\|A e^{t A}\{\hat{\zeta}(\cdot, t)-\hat{\zeta}(\cdot, 0)\}\right\| .
\end{aligned}
$$

Let derive the estimate of $J_{1}(t)$. For $t>r$ we have

$$
\begin{aligned}
\left|G_{2}(\cdot, t)-G_{2}(\cdot, r)\right| \leq & \left|b_{2}\left(\bar{\rho}(\cdot, t), \partial_{\sigma} \bar{\rho}(\cdot, t)\right)-b_{2}\left(\rho_{0}, \partial_{\sigma} \rho_{0}\right)\right|\left|\partial_{\sigma}^{3} \bar{\rho}(\cdot, t)-\partial_{\sigma}^{3} \bar{\rho}(\cdot, r)\right| \\
& +\left|b_{2}\left(\bar{\rho}(\cdot, t), \partial_{\sigma} \bar{\rho}(\cdot, t)\right)-b_{2}\left(\bar{\rho}(\cdot, r), \partial_{\sigma} \bar{\rho}(\cdot, r)\right)\right|\left|\partial_{\sigma}^{3} \bar{\rho}(\cdot, r)\right| \\
& +\left|g_{2}\left(\bar{\rho}(\cdot, t), \partial_{\sigma} \bar{\rho}(\cdot, t), \partial_{\sigma}^{2} \bar{\rho}(\cdot, t)\right)-g_{2}\left(\bar{\rho}(\cdot, r), \partial_{\sigma} \bar{\rho}(\cdot, r), \partial_{\sigma}^{2} \bar{\rho}(\cdot, r)\right)\right| \\
\leq & C_{K}\left\{t^{\frac{1+\alpha}{4}} \cdot r^{-\frac{1}{2}}(t-r)^{\frac{1+\alpha}{4}}+r^{-\frac{1}{2}}(t-r)^{\frac{3+\alpha}{4}} \cdot r^{-\frac{1}{4}}+r^{-\frac{1}{2}}(t-r)^{\frac{2+\alpha}{4}}\right\} .
\end{aligned}
$$

This fact and characterization of interpolation spaces $D_{A}(\beta, \infty)$ imply that

$$
\begin{aligned}
J_{1}(t) \leq & C_{0} \int_{0}^{t}(t-r)^{\frac{3}{4}-2}\left\|\left(\sigma-l_{-}\right)^{3} \eta\right\|_{D_{A}\left(\frac{3}{4}, \infty\right)}\left|G_{2}\left(l_{-}, t\right)-G_{2}\left(l_{-}, r\right)\right| d r \\
\leq & C_{0, K} \int_{0}^{t}(t-r)^{\frac{3}{4}-2}\left\{t^{\frac{1+\alpha}{4}} \cdot r^{-\frac{1}{2}}(t-r)^{\frac{1+\alpha}{4}}\right. \\
& \left.\quad+r^{-\frac{1}{2}}(t-r)^{\frac{3+\alpha}{4}} \cdot r^{-\frac{1}{4}}+r^{-\frac{1}{2}}(t-r)^{\frac{2+\alpha}{4}}\right\} d r \\
\leq & C_{0, K, \alpha}\left(t^{\frac{1+\alpha}{4}}+t^{\frac{1}{4}}+t^{\frac{1}{4}}\right) t^{\frac{\alpha}{4}-\frac{1}{2}} \\
\leq & \widetilde{C}_{0, K, \alpha}\left(t^{\frac{1+\alpha}{4}}+t^{\frac{1}{4}}\right) t^{\frac{\alpha}{4}-\frac{1}{2}} .
\end{aligned}
$$


Applying the similar argument to $J_{2}(t)$, we are led to

$$
\begin{aligned}
J_{2}(t) & \leq C_{0} t^{\frac{3}{4}-1}\left\|\left(\sigma-l_{-}\right)^{3} \eta\right\|_{D_{A}\left(\frac{3}{4}, \infty\right)}\left|G_{2}\left(l_{-}, t\right)-G_{2}\left(l_{-}, 0\right)\right| \\
& \leq C_{0, K} t^{\frac{3}{4}-1}\left(t^{\frac{1+\alpha}{4}} \cdot K t^{-\frac{1}{4}}+t^{\frac{\alpha}{4}}\right) \\
& \leq \widetilde{C}_{0, K} t^{\frac{1}{4}} \cdot t^{\frac{\alpha}{4}-\frac{1}{2}} .
\end{aligned}
$$

Since the estimates for the other terms are also obtained similarly, we have

$$
\|A \dot{z}(t)\| \leq C_{0, K, \alpha} T^{\frac{1}{4}} \cdot t^{\frac{\alpha}{4}-\frac{1}{2}} .
$$

It follows from (B.5) that

$$
I_{1}(t) \leq C_{0, K, \alpha} T^{\frac{1}{4}} \cdot(\varepsilon / 2)^{-\frac{1}{2}} .
$$

Let us derive the estimate for $I_{2}(t)$. Set

$$
w(t):=\int_{\varepsilon / 2}^{t} A^{2} e^{(t-r) A}\{\zeta(\cdot, r)-\zeta(\cdot, t)\} d r .
$$

In order to obtain the estimate of $\|w\|_{D_{A}\left(\frac{\alpha}{4}, \infty\right)}$, we recall the definition of $\|\cdot\|_{D_{A}\left(\frac{\alpha}{4}, \infty\right)}$. Since the estimate of $\|w\|$ is similar to that of $J_{1}(t)$, we consider only the estimate of the semi-norm. According to the definition, we see

$$
\begin{aligned}
{[w]_{D_{A}\left(\frac{\alpha}{4}, \infty\right)} } & =\sup _{0<\tau<1}\left\|\tau^{1-\frac{\alpha}{4}} A e^{\tau A} w\right\| \\
& \leq \sup _{0<\tau<1} \tau^{1-\frac{\alpha}{4}} \int_{\varepsilon / 2}^{t}\left\|A^{3} e^{(t+\tau-r) A}\{\zeta(\cdot, r)-\zeta(\cdot, t)\}\right\| d r
\end{aligned}
$$

We show the estimate only for the term including $\hat{\zeta}(\sigma, t)$. In fact we obtain

$$
\begin{aligned}
& \tau^{1-\frac{\alpha}{4}} \int_{\varepsilon / 2}^{t}\left\|A^{3} e^{(t+\tau-r) A}\{\hat{\zeta}(\cdot, r)-\hat{\zeta}(\cdot, t)\}\right\| d r \\
& \leq C_{0} \tau^{1-\frac{\alpha}{4}} \int_{\varepsilon / 2}^{t}(t+\tau-r)^{\frac{3}{4}-3}\left\|\left(\sigma-l_{-}\right)^{3} \eta\right\|_{D_{A}\left(\frac{3}{4}, \infty\right)}\left|G_{2}\left(l_{-}, t\right)-G_{2}\left(l_{-}, r\right)\right| d r \\
& \leq C_{0, K} \tau^{1-\frac{\alpha}{4}} \int_{\varepsilon / 2}^{t}(t+\tau-r)^{\frac{3}{4}-3}\left\{t^{\frac{1+\alpha}{4}} \cdot(\varepsilon / 2)^{-\frac{1}{2}}(t-r)^{\frac{1+\alpha}{4}}\right. \\
& \left.\quad+(\varepsilon / 2)^{-\frac{1}{2}}(t-r)^{\frac{3+\alpha}{4}} \cdot r^{-\frac{1}{4}}+(\varepsilon / 2)^{-\frac{1}{2}}(t-r)^{\frac{2+\alpha}{4}}\right\} d r \\
& \leq C_{0, K} \tau^{1-\frac{\alpha}{4}} \int_{\varepsilon / 2}^{t}(t+\tau-r)^{\frac{\alpha}{4}-2} d r \cdot\left(t^{\frac{1+\alpha}{4}}+t^{\frac{1}{4}}\right) \cdot(\varepsilon / 2)^{-\frac{1}{2}} \\
& \quad+C_{0, K} \tau^{1-\frac{\alpha}{4}} \int_{\varepsilon / 2}^{t}(t+\tau-r)^{\frac{\alpha}{4}-2}(r-\varepsilon / 2)^{-\frac{1}{4}} d r \cdot(t-\varepsilon / 2)^{\frac{1}{2}} \cdot(\varepsilon / 2)^{-\frac{1}{2}} \\
& \leq C_{0, K, \alpha} \tau^{1-\frac{\alpha}{4}} \cdot \tau^{\frac{\alpha}{4}-1}\left\{T^{\frac{1}{4}}+(t-\varepsilon / 2)^{\frac{1}{4}}\right\} \cdot(\varepsilon / 2)^{-\frac{1}{2}} \\
& \leq C_{0, K, \alpha} T^{\frac{1}{4}} \cdot(\varepsilon / 2)^{-\frac{1}{2}} .
\end{aligned}
$$

As a consequence, we are led to

$$
I_{2}(t) \leq C_{0, K, \alpha} T^{\frac{1}{4}} \cdot(\varepsilon / 2)^{-\frac{1}{2}} .
$$


The estimate of $I_{3}(t)$ is omitted, since we can readily obtain it by using (B.1) together with the estimate of $\left|G_{2}(\cdot, t)-G_{2}(\cdot, r)\right|$.

Consequently, we have

$$
\|A \dot{z}(t)\|_{D_{A}\left(\frac{\alpha}{4}, \infty\right)} \leq C_{0, K, \alpha} T^{\frac{1}{4}} \cdot \varepsilon^{-\frac{1}{2}} \quad \text { for } \quad \varepsilon \leq t \leq T .
$$

This completes the proof of the second estimate of (B.4).

\section{References}

[1] P. Acquistapace, B. Terreni, Hölder classes with boundary conditions as interpolation spaces, Math. Z., 195 (1987), 451-471.

[2] J. W. Barrett, H. Garcke, and R. Nürnberg, A parametric finite element method for fourth order geometric evolution equations, Journal of Computational Physics 222, Issue 1 (2007), 441-467.

[3] J. W. Barrett, H. Garcke, and R. Nürnberg, On the variational approximation of combined second and fourth order geometric evolution equations, SIAM J. Scientific Comput. 29, Issue 3 (2007), 1006-1041.

[4] J. W. Cahn, C. M. Elliott, and A. Novick-Cohen A, The Cahn-Hilliard equation with a concentration dependent mobility: motion by minus the Laplacian of the mean curvature, European J. Appl. Math., 7 (1996), no.3, 287-301.

[5] X. Chen, The Hele-Shaw problem and area-preserving curve-shortening motions, Arch. Rational Mech. Anal. 123 (1993), no. 2, 117-151.

[6] F. Davi and M. Gurtin M, On the motion of a phase interface by surface diffusion, J. of Appl. Math. and Phys. (ZAMP), 41 (1990), 782-811.

[7] C. M. Elliott and H. Garcke, Existence results for diffusive surface motion laws, Adv. Math. Sci. Appl., 7, No.1 (1997), 465-488.

[8] J. Escher, H. Garcke, K. Ito, Exponential stability for a mirror-symmetric three phase boundary motion by surface diffusion, Mathematische Nachrichten 257 (2003), 3-15.

[9] J. Escher, U. F. Mayer, and G. Simonett, The surface diffusion flow for immersed hypersurfaces, SIAM J. Math. Anal., 29, no.6 (1998), 1419-1433.

[10] S. -I. Ei, M. -H. Sato, and E. Yanagida, Stability of stationary interfaces with contact angle in a generalized mean curvature flow, Amer. J. Math., 118 (1996), 653-687.

[11] S. -I. Ei and E. Yanagida, Stability of stationary interfaces in a generalized mean curvature flow, J. Fac. Sci. Univ. Tokyo, Sect. IA, Math., 40 (1993), 651-661.

[12] H. Garcke, K. Ito, and Y. Kohsaka, Linearized stability analysis of stationary solutions for surface diffusion with boundary conditions, SIAM J. Math. Anal., 36 (2005), $1031-1056$ 
[13] K. Ito and Y. Kohsaka, Three phase boundary motion by surface diffusion: Stability of a mirror symmetric stationary solution, Interfaces Free Bound., 3 (2001), 45-80.

[14] K. Ito and Y. Kohsaka, Three phase boundary motion by surface diffusion in triangular domain, Adv. Math. Sci. Appl., 11 (2001), 753-779.

[15] R. Ikota and E. Yanagida, A stability criterion for stationary curves to the curvaturedriven motion with a triple junction, Differential Integral Equations, 16 (2003), 707726.

[16] K. Grosse-Brauckmann, Stable constant mean curvature surfaces minimize area, Pacific J. Math., 175, No. 2 (1996), 527-534.

[17] A. Lunardi, Analytic semigroups and optimal regularity in parabolic problems, Birkhäuser, 1995.

[18] A. Lunardi, E. Sinestrari, and W. Von Wahl, A semigroup approach to the time dependent parabolic initial-boundary value problem, Diff. Int. Eqns., 5 (1992), 12751306.

[19] W. W. Mullins, Theory of thermal grooving, J. Appl. Phys., 28 (1957), 333-339.

[20] H. B. Stewart, Generation of analytic semigroups by strongly elliptic operators under general boundary conditions, Trans. Amer. Math. Soc., 259 (1980), 299-310.

[21] J. E. Taylor and J. W. Cahn, Linking anisotropic sharp and diffuse surface motion laws via gradient flows, J. Stat. Phys. 77, no.1-2 (1994), 183-197.

[22] T. Vogel, Sufficient conditions for capillary surfaces to be energy minima, Pacific J. Math., 194, No. 2 (2000), 469-489.

[23] E. Zeidler, Nonlinear Functional Analysis and its Applications I, Springer Verlag 1986 